\title{
Use of Ra isotopes to deduce rapid transfer of sediment-derived inputs off Kerguelen
}

\author{
V. Sanial ${ }^{1}$, P. van Beek ${ }^{1}$, B. Lansard ${ }^{1,2}$, M. Souhaut ${ }^{1}$, E. Kestenare ${ }^{1}$, F. d'Ovidio ${ }^{3}$, M. Zhou ${ }^{4}$, and S. Blain ${ }^{5,6}$ \\ ${ }^{1}$ LEGOS, Laboratoire d'Etudes en Géophysique et Océanographie Spatiales, (CNRS-CNES-IRD-UPS), Observatoire Midi \\ Pyrénées, 14 avenue Edouard Belin, 31400 Toulouse, France \\ ${ }^{2}$ LSCE, Laboratoire des Sciences du Climat et de l'Environnement (IPSL-CEA-CNRS-UVSQ-IPSL), avenue de la Terrasse, \\ 91198 Gif-sur-Yvette, France \\ ${ }^{3}$ Sorbonne Universités, UPMC Univ Paris 06, CNRS, LOCEAN-IPSL, 4 place Jussieu, 75005 Paris, France \\ ${ }^{4}$ Department of Environment Earth and Ocean Sciences, University of Massachusetts Boston, Boston, Massachusetts, USA \\ ${ }^{5}$ Sorbonne Universités, UPMC Univ Paris 06, UMR7621, Laboratoire d'Océanographie Microbienne, Observatoire \\ Océanologie, 66650 Banyuls/mer, France \\ ${ }^{6}$ CNRS, UMR7621, Laboratoire d'Océanologie Microbienne, Observatoire Océanologie, 66650 Banyuls/mer, France
}

Correspondence to: V. Sanial (virginie.sanial@legos.obs-mip.fr)

Received: 5 September 2014 - Published in Biogeosciences Discuss.: 1 October 2014

Revised: 12 January 2015 - Accepted: 13 January 2015 - Published: 5 March 2015

\begin{abstract}
The Southern Ocean is known to be the largest high-nutrient, low-chlorophyll (HNLC) region of the global ocean due to iron limitation. However, a large phytoplankton bloom develops annually downstream of the Kerguelen Islands, a bloom which is sustained partly by iron released from the sediments deposited onto the shelves. In the framework of the KEOPS- 2 project, we used radium isotopes $\left({ }^{224} \mathrm{Ra}, T_{1 / 2}=3.66 \mathrm{~d} ;{ }^{223} \mathrm{Ra}, T_{1 / 2}=11.4 \mathrm{~d} ;{ }^{228} \mathrm{Ra}\right.$, $T_{1 / 2}=5.75 \mathrm{yr}$ ) to provide information on the origin of iron fertilization and on the timescales of the transfer of sedimentderived inputs (including iron and other micronutrients) towards offshore waters. Significant ${ }^{224} \mathrm{Ra}$ and ${ }^{223} \mathrm{Ra}$ activities were found in the near vicinity of the Kerguelen Islands, in agreement with the short half-lives of these isotopes. Significant ${ }^{224} \mathrm{Ra}$ and ${ }^{223} \mathrm{Ra}$ activities were also detected up to $200 \mathrm{~km}$ downstream of the islands and more unexpectedly in offshore waters south of the polar front. These observations thus clearly indicate (i) that the sediment-derived inputs are rapidly transferred towards offshore waters (on timescales on the order of several days up to several weeks) and (ii) that the polar front is not a physical barrier for the chemical elements released from the sediments of the Kerguelen Plateau. The Ra data set suggests that iron and other micronutrients released by the shelves of the Kerguelen Islands may contribute to fueling the phytoplankton bloom downstream of
\end{abstract}

the islands, despite the presence of the polar front. However, the heterogeneous distribution of the ${ }^{224} \mathrm{Ra}$ and ${ }^{223} \mathrm{Ra}$ activities in surface waters suggests that this supply across the front is not a continuous process but rather a process that is highly variable in space and time.

\section{Introduction}

The Southern Ocean is recognized as the major high-nutrient, low-chlorophyll (HNLC) region of the global ocean. Despite high nutrient concentrations, the phytoplankton growth was shown to be limited by the very low iron concentrations in surface waters of the Southern Ocean (De Baar et al., 1995; Martin et al., 1990). Dissolved iron is, however, supplied to the surface waters in several locations of the Southern Ocean where iron is released by the shelf sediments, but this natural iron fertilization remains spatially limited (Tagliabue et al., 2014). Consequently, high phytoplankton biomass can be found offshore the Antarctic continental shelf (Arrigo et al., 2008; Moore and Abbott, 2002) or in the vicinity of subantarctic islands (Blain et al., 2001; Korb et al., 2004; Pollard et al., 2007).

One of the largest phytoplankton blooms is observed offshore of the Kerguelen Islands, in the Indian sector of the 
Southern Ocean (Blain et al., 2001). This phytoplankton bloom extends more than $1000 \mathrm{~km}$ downstream of the Kerguelen Islands and shows two main features: (i) a plume that extends northeast from the islands and north of the polar front (PF) that shows high mesoscale and temporal variability, and (ii) a larger bloom to the southeast of the islands and south of the PF (Blain et al., 2001, 2007). The two areas are separated by a narrow band of relatively low chlorophyll concentration associated with the PF that follows the inner shelf edge between 200 and $500 \mathrm{~m}$ isobaths (Park and Gamberoni, 1997; Park et al., 1998b). While Park et al. (2008a) suggest that the northward geostrophic flow associated with the PF may possibly block any southward penetration of lithogenic inputs released by the Kerguelen Islands, the numerous eddies and meanders formed along the PF may contribute to transport of chemical elements between the northern Kerguelen Plateau and offshore waters.

The KEOPS-2 (KErguelen Ocean and Plateau compared Study) project aimed at understanding the circulation patterns off the Kerguelen Islands and the mechanisms of iron fertilization in that area. The KEOPS- 2 cruise was conducted during austral spring 2011 to the east of the Kerguelen Islands. Natural radio-tracers such as radium isotopes $\left({ }^{223} \mathrm{Ra}\right.$, $\left.T_{1 / 2}=11.4 \mathrm{~d} ;{ }^{224} \mathrm{Ra}, T_{1 / 2}=3.66 \mathrm{~d} ;{ }^{228} \mathrm{Ra}, T_{1 / 2}=5.75 \mathrm{yr}\right)$ have already been proved to be powerful tools to track the origin and fate of chemical elements - including iron and other micronutrients - which are released by the sediments deposited on the shelves (Annett et al., 2013; van Beek et al., 2008; Charette et al., 2007; Dulaiova et al., 2009; Sanial et al., 2014). In this work, we refer to these latter inputs as "sediment-derived inputs". Radium isotopes are produced by the decay of particle-bound thorium isotopes in the sediments and are delivered to the open ocean by diffusion and advection processes. Thus, a water mass that interacts with shelf sediments is potentially enriched in radium and in other elements that also diffuse out of the sediments (e.g., iron and other micronutrients). While iron may then be removed from the water column by biotic or abiotic processes, radium behaves as a conservative tracer. Radium is only affected by radioactive decay and mixing in such a way that the water body keeps the signature of its contact with the sediments. The radium signature of a given water mass may then be transferred by diffusion and advection towards offshore waters. The presence of significant $\mathrm{Ra}$ activities in offshore waters thus indicates that the water body has interacted with shallow sediments. Alternatively, vertical mixing may also transport Ra towards surface waters. Because radium isotopes decay, they can be used as chronometers to estimate the time elapsed since the water body left the shelf, which in turn gives information on how quickly the microelements released by the shallow sediments may be transferred to offshore waters (Moore, 2000). In this work, we examined the distribution of ${ }^{223} \mathrm{Ra},{ }^{224} \mathrm{Ra}$ and ${ }^{228} \mathrm{Ra}$ in surface waters downstream of the Kerguelen Islands in order (i) to investigate the origin and dispersion of the sediment-derived inputs, including iron, and (ii) to determine the apparent ages of offshore waters that provide information on the timescales of the transfer of water and associated chemical elements between the shelves and offshore waters. In addition to the Ra distribution in surface waters, we report several vertical profiles of ${ }^{223} \mathrm{Ra},{ }^{224} \mathrm{Ra}$ and ${ }^{228} \mathrm{Ra}$ that provide constraints on the vertical transport of chemical elements associated with vertical mixing.

\section{Material and methods}

\subsection{The KEOPS-2 project}

The KEOPS-2 cruise took place east of the Kerguelen Islands (northern Kerguelen Plateau) between 14 October and 23 November 2011 onboard the R/V Marion Dufresne (IPEV: Institut Polaire Français - Paul Emile Victor; TAAF: Terres Australes et Antarctiques Francaises). The KEOPS-2 project was designed to study the mechanisms of natural iron fertilization downstream of the Kerguelen Islands and its impact on ecosystems and biogeochemical cycles. The KEOPS2 project was labeled as a GEOTRACES process study and followed up a first KEOPS project conducted in 2005 in the area of the southern Kerguelen Plateau (Blain et al., 2007).

\subsection{Study area}

The Kerguelen Plateau, located in the Indian sector of the Southern Ocean, constitutes one of the few physical barriers for the eastward-flowing Antarctic Circumpolar Current (ACC). Various studies provide a detailed description of the general ocean circulation patterns around the Kerguelen Plateau (Charrassin et al., 2004; Park and Gambéroni, 1995; Park et al., 1998, 2008b, 2009). An important oceanographic feature of the area is the presence of the PF, which is commonly characterized by the northernmost position of a subsurface temperature minimum bounded by the $2{ }^{\circ} \mathrm{C}$ isotherm (Belkin and Gordon, 1996; Park and Gamberoni, 1997; Park et al., 1993). A strong eastward current associated with this front is deflected to the north at $71^{\circ} \mathrm{E}$ following the eastern shelf slope of the Kerguelen Plateau between the 200 and $500 \mathrm{~m}$ isobaths and forms a cyclonic meander that turns southward at $75^{\circ} \mathrm{E}$ (Belkin and Gordon, 1996; Orsi et al., 1995; Park and Gamberoni, 1997; Park et al., 1993; Pollard et al., 2002). Numerous eddies are generated along the PF to the east of the Kerguelen Plateau that can, in some cases, be identified on the satellite composite images of sea surface chlorophyll. The locations of the stations investigated in this study are shown in Fig. 1. 


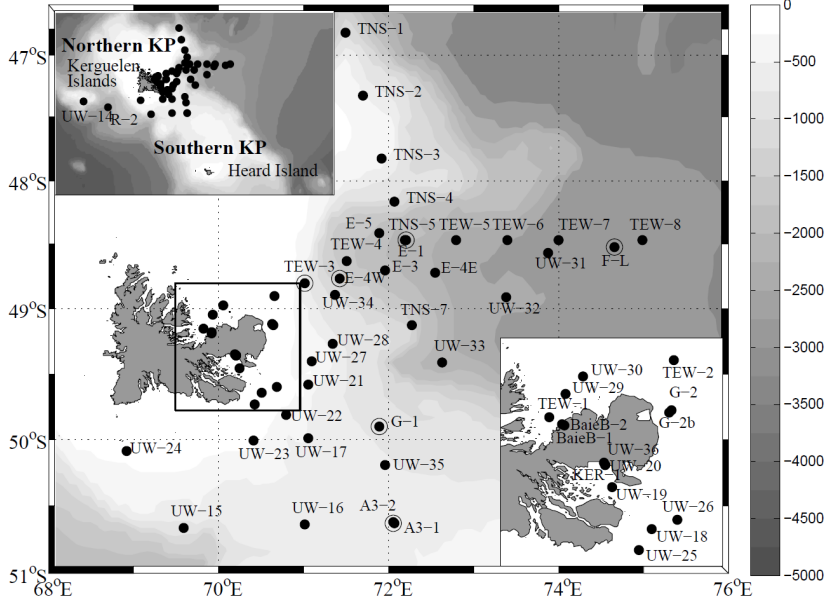

Figure 1. Location of stations investigated for Ra analysis. Solid circles represent surface seawater samples. Circles show the locations where vertical profiles were made. KP is the abbreviation for Kerguelen Plateau. Gray shading represents depth in meters.

\subsection{Radium data}

\subsubsection{Sample collection}

Surface seawater samples were collected at $7 \mathrm{~m}$ depth using a clean pump specially designed by IPEV for the KEOPS2 cruise. Large volumes of surface seawater were collected (250-900 L) and stored in large plastic tanks. We used a CTD (SBE-19plus, Sea-Bird ${ }^{\circledR}$ ) and a rosette system equipped with $22 \times 12 \mathrm{~L}$ Niskin bottles to collect seawater samples from various depths throughout the water column. Three samples were also collected directly on two beaches of the Kerguelen Islands (Baie du Morbihan: samples KER-1; Baie des Baleiniers: samples BaieB-1 and BaieB-2). Seawater samples were then passed by gravity through PVC cartridges filled with "Mn fibers" $\left(\mathrm{MnO}_{2}\right.$-impregnated acrylic fiber) following Moore (2008). The flow rate was fixed at $\leq 0.5 \mathrm{~L} \mathrm{~min}^{-1}$ to provide $100 \%$ extraction efficiency (Moore, 2008; van Beek et al., 2010). The Mn fibers were then rinsed with Milli-Q water and partially dried before analysis.

\subsubsection{Sample analysis}

The Mn fibers were analyzed using a radium delayed coincidence counter (RaDeCC; Charette et al., 2001; Moore and Arnold, 1996; Moore, 2008). Three counting sessions are necessary to determine both excess ${ }^{224} \mathrm{Ra}$ and excess ${ }^{223} \mathrm{Ra}$ activities in the samples. The first counting was performed onboard the research vessel during the cruise and provides the total ${ }^{224} \mathrm{Ra}$ and ${ }^{223} \mathrm{Ra}$ activities. The Mn fibers were analyzed again 3 weeks after sampling to determine the ${ }^{224} \mathrm{Ra}$ activities supported by ${ }^{228} \mathrm{Th}$ and then after 3 months to determine the ${ }^{223} \mathrm{Ra}$ activities supported by ${ }^{227} \mathrm{Ac}$ (Moore,
2000). The ${ }^{224} \mathrm{Ra}$ activities are corrected for the ${ }^{224} \mathrm{Ra}$ supported by ${ }^{228} \mathrm{Th}$ and the ${ }^{223} \mathrm{Ra}$ activities are corrected for the ${ }^{223} \mathrm{Ra}$ supported by ${ }^{227} \mathrm{Ac}$. The ${ }^{224} \mathrm{Ra}$ and ${ }^{223} \mathrm{Ra}$ activities discussed hereafter thus refer to these excess ${ }^{224} \mathrm{Ra}$ and ${ }^{223} \mathrm{Ra}$ activities. Uncertainties for both isotopes were calculated following Garcia-Solsona et al. (2008) and were reported with a $1 \sigma$ confidence interval.

Activities of ${ }^{228} \mathrm{Ra}$ were then determined using the lowbackground gamma detectors placed at the LAFARA underground laboratory in the French Pyrénées (van Beek et al., 2010, 2013). Mn fibers were either ashed at $820^{\circ} \mathrm{C}$ for $16 \mathrm{~h}$ (Charette et al., 2001) and analyzed using a well-type germanium detector or compressed and analyzed using a semiplanar detector. Cross-calibrations between the two detectors were made to avoid any bias in the determination of the Ra activities. Each sample was analyzed for ca. $120 \mathrm{~h}$ to allow for the quantification of the low ${ }^{228} \mathrm{Ra}$ activities present in Southern Ocean waters (Kaufman et al., 1973). ${ }^{228} \mathrm{Ra}$ activities were determined using ${ }^{228} \mathrm{Ac}$ peaks $(338,911$ and $969 \mathrm{keV})$. All radium activities are reported in disintegration per minute per $100 \mathrm{~L}$ of seawater $\left(\mathrm{dpm} 100 \mathrm{~L}^{-1}\right)$. The uncertainties reported for gamma counting consist in the error associated with counting statistics $(1 \sigma)$.

\subsection{Physical data}

\subsubsection{Color data}

High-resolution maps $\left(1 / 25^{\circ} \times 1 / 25^{\circ}\right)$ of chlorophyll concentration $\left(\mathrm{mg} \mathrm{m}^{-3}\right)$ were constructed by a 10-day weighted mean of MODIS and MERIS measurements. These satellite products were delivered 3 times a week in near-real time during the cruise from Ssalto/Duacs and CLS (Collecte Localisation Satellites, Toulouse, France) with support from CNES (Centre National d'Etudes Spatiales, France). These images were used to define the sampling strategy in the investigated area.

\subsubsection{Surface drifters}

Drifters provided by the US National Ocean and Atmospheric Administration (NOAA) Global Drifter Program (GDP) were also released. The drogue is centered at $30 \mathrm{~m}$ depth. These drifters thus provide information on the mean currents in the surface mixed layer and on the dispersion of water masses due to eddy activities. Successive positions of the drifter were transmitted to the R/V Marion Dufresne four times a day by the NOAA GDP center. The time-irregular positions of the drifter were interpolated into a regular time step of $12 \mathrm{~min}$ and a low-pass filter of $48 \mathrm{~h}$ was then applied to filter all tidal currents and inertial oscillations.

\subsubsection{Lagrangian particle analysis}

The Lagrangian particle analysis was based on total surface currents, which are the sum of the absolute geostrophic 
currents (deduced from altimeter product) and Ekman currents (daily mean). The Ekman component is deduced from the European Centre for Medium-Range Weather Forecasts (ECMWF) wind stress analysis applying a regional Ekman model, specifically adjusted for the Kerguelen area. The altimeter current products were produced by Ssalto/Duacs and distributed by AVISO, with support from CNES. Total surface currents were delivered every day with a $1 / 8^{\circ} \times 1 / 8^{\circ}$ resolution. Details of the mapping technique are given by Dibarboure et al. (2011).

\subsubsection{Lagrangian model}

The altimetry-derived velocities providing the geostrophic mesoscale velocity at the ocean surface were analyzed in near-real time with a Lagrangian model. This model was inspired by Mongin et al. (2009), who reconstructed the extension of the Kerguelen chlorophyll plume with a transport scheme based on altimetry. The model created thousands of virtual surface drifters released on the shelf break of Kerguelen $(2000 \mathrm{~m}$ isobaths; apparent age $=0)$. The trajectories were constructed by backward-in-time integration of the altimetric velocity field and were stopped when a hit over the Kerguelen shelf break was detected (indicating a trajectory coming from the shelf) or when a maximum integration time - set to 120 days - was reached (indicating no interaction with the shelf on the past 120 days). This model was applied successfully by Sanial et al. (2014) to highlight the key role played by surface horizontal transport in defining the extension of the springtime chlorophyll plume in the Crozet area.

\section{Results}

\subsection{Hydrological context during the KEOPS-2 cruise}

The KEOPS- 2 cruise lasted almost 2 months (OctoberNovember 2011). During that period, the phytoplankton bloom developed off the Kerguelen Islands (Fig. 2). The satellite composite images of sea surface chlorophyll reveal a complex shape of the phytoplankton bloom that may be associated with the complex hydrography of the area. A high concentration of chlorophyll first appeared close to the Kerguelen Islands (October 2011) before spreading out in offshore waters until covering a large part of the study area at the end of November 2011. East of the Kerguelen Islands, a narrow band of low chlorophyll concentration is associated with the northward branch of the PF that splits the phytoplankton bloom into two parts.

The PF also delimits two surface water masses characterized by a strong contrast in temperature and salinity; the Antarctic Surface Water (AASW) is located south of the PF, and the Subantarctic Surface Water (SASW) is located north of the PF (Emery and Meincke, 1986). The potentialtemperature-salinity diagrams of the water masses investigated in this study are shown in Fig. 3. The SASW is iden- tified only at station F-L, suggesting that this station is located north of the PF. The Winter Water (WW), a typical feature of the Antarctic zone, is the winter remnant of the Antarctic Surface Water characterized by a subsurface temperature minimum layer around $200 \mathrm{~m}$ depth (Park et al., 1998a, 2008b, 2014). The WW is found on all the vertical profiles reported here except for station F-L, thus confirming its location north of the PF. Below the WW, three water masses can be identified: the Upper Circumpolar Deep Water (UCDW), the Lower Circumpolar Deep Water (LCDW) and the Antarctic Bottom Water (AABW) (Park et al., 1993, $2008 \mathrm{~b}$ ). Note that the AABW is only found on the F-L profile (commonly observed below $2600 \mathrm{~m}$ in this area; Park et al., 2008b).

\subsection{Radium distribution in surface waters}

The radium activities reported in this study are shown in Table 1 and fall in the range of previous radium data reported for surface waters near islands of the Southern Ocean (Annett et al., 2013; Charette et al., 2007; Dulaiova et al., 2009; Hanfland, 2002; Kaufman et al., 1973; Sanial et al., 2014; van Beek et al., 2008). The highest ${ }^{223} \mathrm{Ra},{ }^{224} \mathrm{Ra}$ and ${ }^{228} \mathrm{Ra}$ activities are found in seawater samples collected at shallow stations near the Kerguelen Islands (bathymetry $<200 \mathrm{~m}$; Fig. 4). The radium activities then gradually decrease offshore. Several samples, however, display significant ${ }^{224} \mathrm{Ra}$ activities in samples collected offshore (Fig. 4a): stations UW-21-23-34 and TEW-7 located along the PF; stations UW-32, E-1 and TEW-5 south of the PF; and station TNS-1 north of the PF. A greater number of offshore stations exhibit significant ${ }^{223} \mathrm{Ra}$ activities, which agrees with the longer half-life of the ${ }^{223} \mathrm{Ra}$ isotope (Fig. 4b). The stations displaying significant ${ }^{224} \mathrm{Ra}$ activities also display significant ${ }^{223} \mathrm{Ra}$ activities. The radium activities are especially high at station TNS-2, located north of the PF, and at stations E-1 and G-1, located south of the PF. Station G-2 was visited twice and showed high ${ }^{223} \mathrm{Ra}$ and ${ }^{224} \mathrm{Ra}$ activities on both visits. Station A3 located on the southern Kerguelen Plateau was also visited twice. Significant ${ }^{223} \mathrm{Ra}$ and ${ }^{224} \mathrm{Ra}$ activities were determined in the water sample collected at station A3-1 during the first visit at station A3 (note, however, that these activities are low) but were both below the detection limit at station A3-2 (second visit at station A3). In contrast, the ${ }^{228} \mathrm{Ra}$ activities are similar for the two visits to A3 (Table 1). All surface samples display significant ${ }^{228} \mathrm{Ra}$ activities up to ca. $300 \mathrm{~km}$ offshore from the Kerguelen Islands (i.e., station TEW-8). Relatively high values are observed at stations TNS-2 and UW-32, located north and south of the PF, respectively (Fig. 4c). Station R-2, which was chosen as the reference station for typical HNLC waters east of the Kerguelen Islands, shows significant ${ }^{223} \mathrm{Ra},{ }^{224} \mathrm{Ra}$ and ${ }^{228} \mathrm{Ra}$ activities in surface waters. 

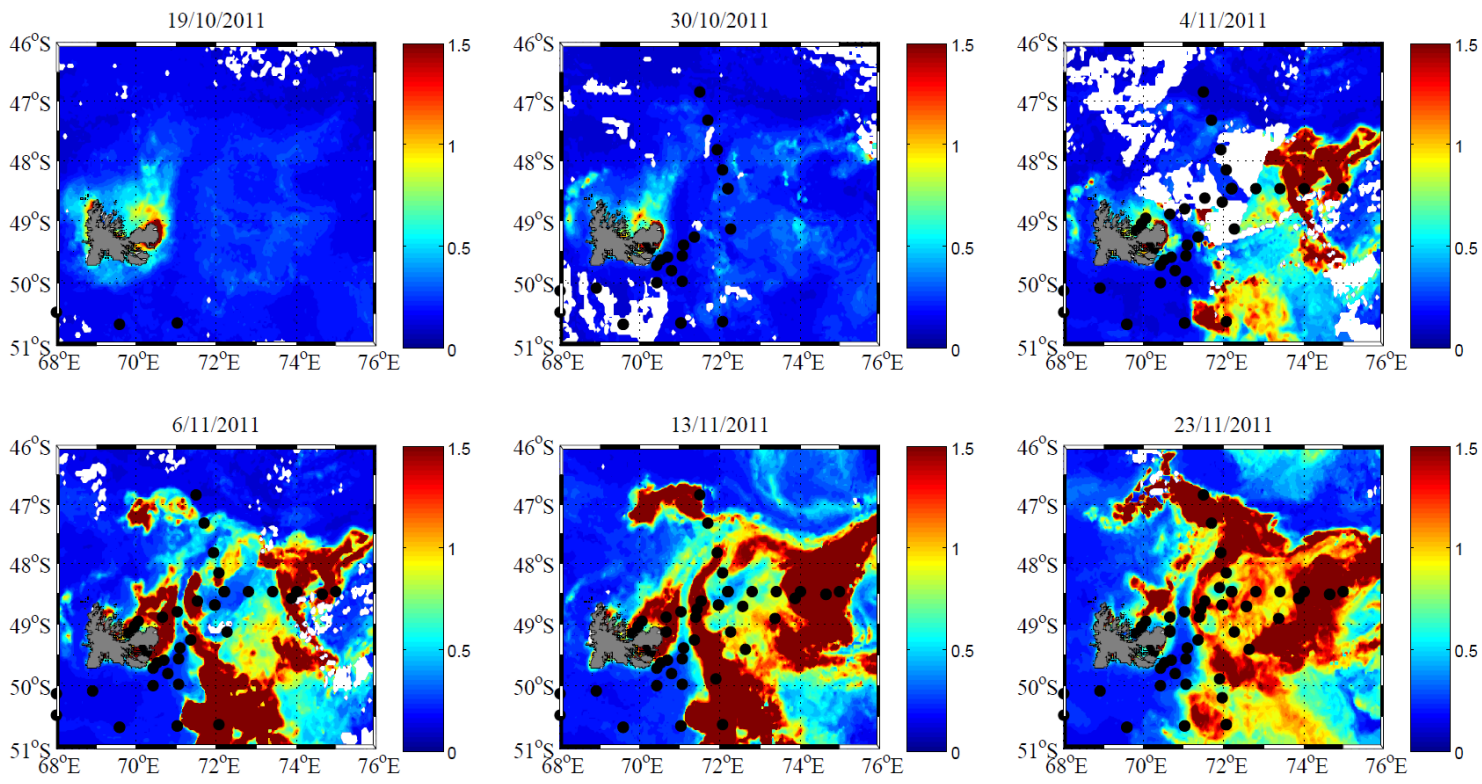

Figure 2. Satellite composite images of sea surface chlorophyll $a\left(\mathrm{mg} \mathrm{m}^{-3}\right)$ at successive dates between the beginning of the KEOPS-2 cruise (19 October 2011) and the end of the cruise (23 November 2011). The locations of the water samples collected for radium analysis within the different time intervals are also reported (solid circles).

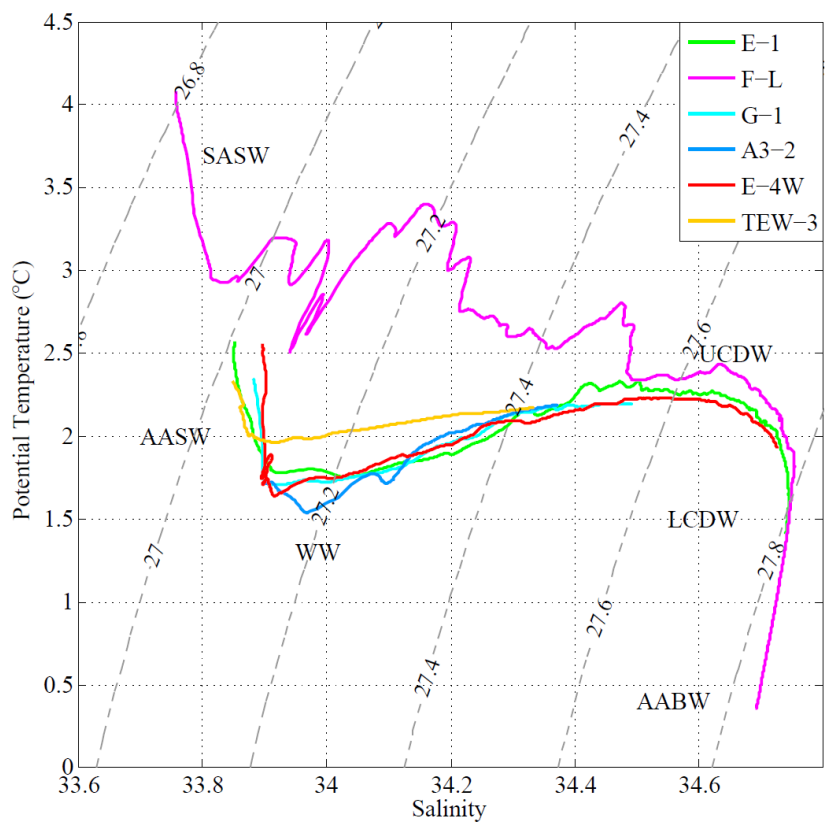

Figure 3. Potential-temperature-salinity diagrams at stations where radium analyses were performed. The main water masses are reported on the figure: Antarctic Surface Water (AASW), Subantarctic Surface Water (SASW), Winter Water (WW), Upper Circumpolar Deep Water (UCDW), Lower Circumpolar Deep Water (LCDW) and Antarctic Bottom Water (AABW).

\subsection{Vertical distribution of Ra isotopes}

The study of the vertical distribution of Ra isotopes allows us to provide constraints on the vertical transport of Ra associated with vertical mixing. Consequently, these profiles help us to define the origin of the Ra enrichments observed in surface waters off the Kerguelen Islands (lateral versus vertical supply of Ra). The major water masses identified with the potential-temperature-salinity diagrams throughout the water column are reported for each profile. The shallow Ra profiles (stations TEW-3, G-1 and A3-2) are shown in Fig. 5 and the deep profiles (stations F-L, E-4W and E-1) are shown in Fig. 6.

The ${ }^{223} \mathrm{Ra}$ and ${ }^{224} \mathrm{Ra}$ activities are usually higher in samples collected near the seafloor and are below the detection limit at intermediate depths (Table 2; Figs. 5 and 6). Significant ${ }^{223} \mathrm{Ra}$ and ${ }^{224} \mathrm{Ra}$ activities are observed in surface and/or subsurface waters several kilometers offshore from the islands, in particular at stations G-1 and E-1, located south of the PF, and at station F-L, located north of the PF. The vertical profiles of ${ }^{223} \mathrm{Ra}$ and ${ }^{224} \mathrm{Ra}$ are quite unique at station F-L. Although (i) this station is located far from the Kerguelen Islands and (ii) the bottom is at $2670 \mathrm{~m}$ depth, the ${ }^{223} \mathrm{Ra}$ and ${ }^{224} \mathrm{Ra}$ activities are relatively high throughout the water column (Fig. 6). Significant ${ }^{228} \mathrm{Ra}$ activities were found in the different water columns investigated in this study. The ${ }^{228} \mathrm{Ra}$ activities at stations TEW- 3 and G- 1 are relatively high and uniform throughout the water column. The ${ }^{228} \mathrm{Ra}$ activities at station A3 are uniform in the upper $250 \mathrm{~m}$ and then increase with increasing water depth. The vertical ${ }^{228} \mathrm{Ra}$ profiles at the deep stations (F-L, E-1 and E-4W) exhibit an in- 
Table 1. Dissolved ${ }^{223} \mathrm{Ra},{ }^{224} \mathrm{Ra}$ and ${ }^{228} \mathrm{Ra}$ activities determined in surface samples collected off the Kerguelen Islands. The ${ }^{223} \mathrm{Ra}$ and ${ }^{224} \mathrm{Ra}$ activities are excess radium activities (see Methods for details). Activities are expressed in disintegration per minute per $100 \mathrm{~L}\left(\mathrm{dpm} 100 \mathrm{~L}^{-1}\right)$. $<$ DL denotes below the detection limit.

\begin{tabular}{|c|c|c|c|c|c|c|c|c|c|c|}
\hline Station & $\begin{array}{l}\text { Sampling } \\
\text { date }\end{array}$ & $\begin{array}{r}\text { Volume } \\
\text { (L) }\end{array}$ & $\begin{array}{r}\text { Depth } \\
(\mathrm{m})\end{array}$ & $\begin{array}{r}\text { Bottom depth } \\
(\mathrm{m})\end{array}$ & $\begin{array}{l}{ }^{223} \mathrm{Ra} \\
\left(\mathrm{dpm} 100 \mathrm{~L}^{-1}\right)\end{array}$ & $\begin{array}{l}{ }^{224} \mathrm{Ra} \\
\left(\mathrm{dpm} 100 \mathrm{~L}^{-1}\right)\end{array}$ & $\begin{array}{l}{ }^{228} \mathrm{Ra} \\
\left(\mathrm{dpm} 100 \mathrm{~L}^{-1}\right)\end{array}$ & ${ }^{223} \mathrm{Ra} /{ }^{228} \mathrm{Ra}$ & ${ }^{224} \mathrm{Ra} /{ }^{228} \mathrm{Ra}$ & ${ }^{224} \mathrm{Ra} /{ }^{223} \mathrm{Ra}$ \\
\hline UW-14 & $17 / 10 / 2011$ & 900 & 7 & 342 & $<\mathrm{DL}$ & $<\mathrm{DL}$ & $0.06 \pm 0.019$ & & & \\
\hline UW-15 & $18 / 10 / 2011$ & 900 & 7 & 712 & $0.008 \pm 0.006$ & $<\mathrm{DL}$ & $0.03 \pm 0.010$ & $0.24 \pm 0.19$ & & \\
\hline UW-16 & $18 / 10 / 2011$ & 900 & 7 & 560 & $0.007 \pm 0.007$ & $<\mathrm{DL}$ & $0.05 \pm 0.013$ & $0.13 \pm 0.13$ & & \\
\hline UW-17 & $19 / 10 / 2011$ & 500 & 7 & 676 & $<\mathrm{DL}$ & $<\mathrm{DL}$ & $0.17 \pm 0.035$ & & & \\
\hline UW-18 & $19 / 10 / 2011$ & 250 & 7 & 104 & $0.062 \pm 0.018$ & $0.213 \pm 0.062$ & $0.49 \pm 0.079$ & $0.13 \pm 0.04$ & $0.44 \pm 0.15$ & $3.45 \pm 1.41$ \\
\hline UW-19 & $19 / 10 / 2011$ & 250 & 7 & 160 & $0.047 \pm 0.016$ & $0.143 \pm 0.052$ & $0.47 \pm 0.139$ & $0.1 \pm 0.05$ & $0.31 \pm 0.14$ & $3.03 \pm 1.51$ \\
\hline UW-20 & $19 / 10 / 2011$ & 250 & 7 & & $0.090 \pm 0.020$ & $0.808 \pm 0.079$ & $1.21 \pm 0.082$ & $0.07 \pm 0.02$ & $0.67 \pm 0.08$ & $8.98 \pm 2.15$ \\
\hline UW-21 & $24 / 10 / 2011$ & 700 & 7 & 597 & $0.011 \pm 0.005$ & $0.036 \pm 0.024$ & $0.11 \pm 0.026$ & $0.1 \pm 0.05$ & $0.33 \pm 0.24$ & $3.38 \pm 2.69$ \\
\hline UW-22 & $24 / 10 / 2011$ & 450 & 7 & 253 & $0.023 \pm 0.007$ & $<\mathrm{DL}$ & $0.20 \pm 0.039$ & $0.11 \pm 0.04$ & & \\
\hline UW-23 & $24 / 10 / 2011$ & 450 & 7 & 233 & $0.008 \pm 0.006$ & $0.116 \pm 0.025$ & $0.04 \pm 0.013$ & $0.19 \pm 0.16$ & $2.92 \pm 1.16$ & $15.17 \pm 11.93$ \\
\hline UW-24 & $24 / 10 / 2011$ & 450 & 7 & 171 & $<\mathrm{DL}$ & $<\mathrm{DL}$ & $0.11 \pm 0.020$ & & & \\
\hline UW-25 & $28 / 10 / 2011$ & 700 & 7 & 116 & $0.060 \pm 0.011$ & $0.218 \pm 0.055$ & $0.08 \pm 0.017$ & $0.72 \pm 0.19$ & $2.62 \pm 0.84$ & $3.64 \pm 1.11$ \\
\hline UW-26 & $28 / 10 / 2011$ & 700 & 7 & 130 & $0.043 \pm 0.010$ & $0.065 \pm 0.033$ & $0.19 \pm 0.023$ & $0.23 \pm 0.06$ & $0.34 \pm 0.18$ & $1.5 \pm 0.84$ \\
\hline UW-27 & $28 / 10 / 2011$ & 500 & 7 & 393 & $0.019 \pm 0.007$ & $<\mathrm{DL}$ & $0.06 \pm 0.016$ & $0.31 \pm 0.14$ & & \\
\hline UW-28 & $28 / 10 / 2011$ & 700 & 7 & 650 & $0.020 \pm 0.008$ & $<\mathrm{DL}$ & $0.07 \pm 0.015$ & $0.29 \pm 0.13$ & & \\
\hline UW-29 & $31 / 10 / 2011$ & 450 & 7 & 100 & $0.031 \pm 0.011$ & $0.118 \pm 0.045$ & $0.53 \pm 0.038$ & $0.06 \pm 0.02$ & $0.22 \pm 0.09$ & $3.81 \pm 2.02$ \\
\hline UW-30 & $31 / 10 / 2011$ & 450 & 7 & 100 & $0.083 \pm 0.014$ & $0.201 \pm 0.039$ & $0.58 \pm 0.039$ & $0.14 \pm 0.03$ & $0.35 \pm 0.07$ & $2.44 \pm 0.62$ \\
\hline UW-31 & $05 / 11 / 2011$ & 700 & 7 & & $<\mathrm{DL}$ & $<\mathrm{DL}$ & $0.17 \pm 0.027$ & & & \\
\hline UW-32 & $08 / 11 / 2011$ & 700 & 7 & 4561 & $0.013 \pm 0.008$ & $0.035 \pm 0.046$ & $0.24 \pm 0.040$ & $0.05 \pm 0.04$ & $0.15 \pm 0.19$ & $2.82 \pm 4.1$ \\
\hline UW-33 & $08 / 11 / 2011$ & 450 & 7 & 1664 & $<\mathrm{DL}$ & $<\mathrm{DL}$ & $0.09 \pm 0.030$ & & & \\
\hline UW-34 & $09 / 11 / 2011$ & 500 & 7 & 1118 & $0.017 \pm 0.011$ & $0.124 \pm 0.078$ & $0.10 \pm 0.020$ & $0.16 \pm 0.12$ & $1.23 \pm 0.8$ & $7.45 \pm 6.84$ \\
\hline UW-35 & $17 / 11 / 2011$ & 700 & 7 & 554 & $<\mathrm{DL}$ & $<\mathrm{DL}$ & $0.16 \pm 0.026$ & & & \\
\hline UW-36 & $21 / 11 / 2011$ & 500 & 7 & 21 & $0.098 \pm 0.016$ & $0.411 \pm 0.098$ & $1.07 \pm 0.054$ & $0.09 \pm 0.02$ & $0.38 \pm 0.09$ & $4.18 \pm 1.22$ \\
\hline UW-37 & $22 / 11 / 2011$ & 900 & 7 & 3720 & $<\mathrm{DL}$ & $<\mathrm{DL}$ & $0.13 \pm 0.016$ & & & \\
\hline TNS-1 & $23 / 10 / 2011$ & 500 & 7 & 2280 & $0.015 \pm 0.005$ & $0.046 \pm 0.033$ & $0.07 \pm 0.022$ & $0.21 \pm 0.1$ & $0.65 \pm 0.5$ & $3.11 \pm 2.46$ \\
\hline TNS-2 & $23 / 10 / 2011$ & 700 & 7 & 520 & $0.023 \pm 0.006$ & $<\mathrm{DL}$ & $0.31 \pm 0.064$ & $0.07 \pm 0.02$ & & \\
\hline TNS-3 & $23 / 10 / 2011$ & 700 & 7 & 540 & $0.006 \pm 0.004$ & $<\mathrm{DL}$ & $0.15 \pm 0.050$ & $0.04 \pm 0.03$ & & \\
\hline TNS-4 & $22 / 10 / 2011$ & 700 & 7 & 1800 & $0.015 \pm 0.005$ & $<\mathrm{DL}$ & $0.08 \pm 0.017$ & $0.2 \pm 0.08$ & & \\
\hline TNS-5 & $22 / 10 / 2011$ & 700 & 7 & 2060 & $0.021 \pm 0.005$ & $0.070 \pm 0.030$ & $0.17 \pm 0.050$ & $0.13 \pm 0.05$ & $0.42 \pm 0.22$ & $3.26 \pm 1.62$ \\
\hline TNS-7 & $21 / 10 / 2011$ & 700 & 7 & 1864 & $<\mathrm{DL}$ & $<\mathrm{DL}$ & $0.03 \pm 0.012$ & & & \\
\hline TEW-1 & $31 / 10 / 2011$ & 700 & 7 & 92 & $0.014 \pm 0.011$ & $0.131 \pm 0.045$ & $0.84 \pm 0.051$ & $0.02 \pm 0.01$ & $0.16 \pm 0.05$ & $9.31 \pm 7.86$ \\
\hline TEW-2 & $31 / 10 / 2011$ & 450 & 7 & 85 & $0.039 \pm 0.011$ & $0.153 \pm 0.045$ & $0.28 \pm 0.027$ & $0.14 \pm 0.04$ & $0.55 \pm 0.17$ & $3.89 \pm 1.56$ \\
\hline TEW-3 & $31 / 10 / 2011$ & 500 & 7 & 557 & $0.008 \pm 0.007$ & $<\mathrm{DL}$ & $0.10 \pm 0.018$ & $0.08 \pm 0.07$ & & \\
\hline TEW-4 & $01 / 11 / 2011$ & 500 & 7 & 1596 & $<\mathrm{DL}$ & $<\mathrm{DL}$ & $0.08 \pm 0.025$ & & & \\
\hline TEW-5 & $01 / 11 / 2011$ & 450 & 7 & 2290 & $<\mathrm{DL}$ & $0.128 \pm 0.044$ & $0.18 \pm 0.033$ & & $0.71 \pm 0.28$ & \\
\hline TEW-6 & $02 / 11 / 2011$ & 450 & 7 & 2400 & $0.011 \pm 0.006$ & $<\mathrm{DL}$ & $0.08 \pm 0.019$ & $0.13 \pm 0.08$ & & \\
\hline TEW-7 & $02 / 11 / 2011$ & 700 & 7 & 2510 & $0.020 \pm 0.009$ & $0.147 \pm 0.062$ & $0.16 \pm 0.019$ & $0.13 \pm 0.06$ & $0.93 \pm 0.4$ & $7.37 \pm 4.51$ \\
\hline TEW-8 & $02 / 11 / 2011$ & 900 & 7 & 2800 & $<\mathrm{DL}$ & $<\mathrm{DL}$ & $0.17 \pm 0.024$ & & & \\
\hline E-1 & $29 / 10 / 2011$ & 900 & 7 & 2065 & $0.021 \pm 0.005$ & $0.070 \pm 0.022$ & $0.10 \pm 0.022$ & $0.23 \pm 0.08$ & $0.73 \pm 0.28$ & $3.26 \pm 1.29$ \\
\hline E-3 & $03 / 11 / 2011$ & 900 & 7 & 1915 & $0.009 \pm 0.005$ & $<\mathrm{DL}$ & $0.03 \pm 0.016$ & $0.33 \pm 0.25$ & & \\
\hline E-4W & $12 / 11 / 2011$ & 900 & 7 & 1385 & $0.01 \pm 0.007$ & $<\mathrm{DL}$ & $0.14 \pm 0.034$ & & & \\
\hline E-4E & $12 / 11 / 2011$ & 500 & 7 & 2210 & $<\mathrm{DL}$ & $<\mathrm{DL}$ & $0.12 \pm 0.021$ & & & \\
\hline E-5 & $18 / 11 / 2011$ & 900 & 7 & 1920 & $<\mathrm{DL}$ & $<\mathrm{DL}$ & $0.11 \pm 0.008$ & & & \\
\hline A3-1 & $19 / 10 / 2011$ & 900 & 7 & 528 & $0.015 \pm 0.003$ & $0.034 \pm 0.024$ & $0.12 \pm 0.041$ & $0.12 \pm 0.05$ & $0.28 \pm 0.21$ & $2.26 \pm 1.62$ \\
\hline A3-2 & $16 / 11 / 2011$ & 900 & 7 & 531 & $<\mathrm{DL}$ & $<\mathrm{DL}$ & $0.12 \pm 0.024$ & & & \\
\hline G-1 & $09 / 11 / 2011$ & 900 & 7 & 592 & $0.023 \pm 0.007$ & $<\mathrm{DL}$ & $0.05 \pm 0.020$ & $0.46 \pm 0.23$ & & \\
\hline G-2 & $09 / 11 / 2011$ & 500 & 7 & 67 & $0.089 \pm 0.015$ & $0.412 \pm 0.052$ & $0.36 \pm 0.046$ & $0.25 \pm 0.05$ & $1.15 \pm 0.21$ & $4.64 \pm 0.98$ \\
\hline $\mathrm{G}-2 \mathrm{~b}$ & $21 / 11 / 2011$ & 500 & 7 & 67 & $0.130 \pm 0.017$ & $0.568 \pm 0.067$ & $0.75 \pm 0.066$ & $0.17 \pm 0.03$ & $0.75 \pm 0.11$ & $4.36 \pm 0.77$ \\
\hline F-L & $06 / 11 / 2011$ & 900 & 7 & 2670 & $<\mathrm{DL}$ & $<\mathrm{DL}$ & $0.17 \pm 0.021$ & & & \\
\hline $\mathrm{R}-2$ & $26 / 10 / 2011$ & 900 & 7 & 2531 & $0.016 \pm 0.009$ & $0.057 \pm 0.028$ & $0.11 \pm 0.016$ & $0.15 \pm 0.09$ & $0.53 \pm 0.27$ & $3.49 \pm 2.51$ \\
\hline KER-1 & $19 / 10 / 2011$ & 87.8 & 1 & 3 & $0.302 \pm 0.048$ & $2.053 \pm 0.125$ & $1.62 \pm 0.160$ & $0.19 \pm 0.03$ & $1.27 \pm 0.15$ & $6.8 \pm 1.16$ \\
\hline BaieB-1 & $31 / 10 / 2011$ & 99.3 & 1 & 3 & $0.219 \pm 0.032$ & $2.332 \pm 0.118$ & $0.88 \pm 0.089$ & $0.25 \pm 0.04$ & $2.65 \pm 0.3$ & $10.67 \pm 1.67$ \\
\hline BaieB-2 & $31 / 10 / 2011$ & 65.2 & 1 & 3 & $0.012 \pm 0.008$ & $0.256 \pm 0.024$ & $2.57 \pm 0.183$ & $0.005 \pm 0.003$ & $0.1 \pm 0.01$ & $20.64 \pm 13.83$ \\
\hline
\end{tabular}

creasing trend with increasing depth reflecting the diffusion of radium out of the sediments. This latter pattern is also especially marked at station A3 on the southern Kerguelen Plateau (Fig. 5).

\section{Discussion}

\subsection{Origin of the radium enrichments in surface waters}

The relatively high radium activities $\left({ }^{223} \mathrm{Ra},{ }^{224} \mathrm{Ra}\right.$ and ${ }^{228} \mathrm{Ra}$ ) observed in surface waters east of the Kerguelen Islands may be explained either by the vertical transport or diffusion that supplies radium to surface waters or by the lateral advection of waters that have recently interacted with shallow sediments (Blain et al., 2001; Park et al., 2008a; van Beek et al., 2008). 
Table 2. Dissolved ${ }^{223} \mathrm{Ra},{ }^{224} \mathrm{Ra}$ and ${ }^{228} \mathrm{Ra}$ activities determined in seawater samples collected in the water column using Niskin bottles. The ${ }^{223} \mathrm{Ra}$ and ${ }^{224} \mathrm{Ra}$ activities are excess radium activities (see Methods for details). Activities are expressed in disintegration per minute per $100 \mathrm{~L}\left(\mathrm{dpm} 100 \mathrm{~L}^{-1}\right)$. The number of counts detected is also reported in the Table $(\mathrm{cnts})$. < DL denotes below the detection limit.

\begin{tabular}{|c|c|c|c|c|c|c|c|c|}
\hline $\begin{array}{l}\text { Station and depth } \\
\text { (m) }\end{array}$ & $\begin{array}{l}\text { Volume } \\
\text { (L) }\end{array}$ & $\begin{array}{r}\text { Bottom depth } \\
(\mathrm{m})\end{array}$ & $\begin{array}{l}{ }^{223} \mathrm{Ra} \\
\left(\mathrm{dpm} 100 \mathrm{~L}^{-1}\right)\end{array}$ & $\begin{array}{r}{ }^{223} \mathrm{Ra} \\
\mathrm{cnts}\end{array}$ & $\begin{array}{l}{ }^{224} \mathrm{Ra} \\
\left(\mathrm{dpm} 100 \mathrm{~L}^{-1}\right)\end{array}$ & $\begin{array}{r}{ }^{224} \mathrm{Ra} \\
\mathrm{cnts}\end{array}$ & $\begin{array}{l}{ }^{228} \mathrm{Ra} \\
\left(\mathrm{dpm} 100 \mathrm{~L}^{-1}\right)\end{array}$ & $\begin{array}{r}{ }^{228} \mathrm{Ra} \\
\mathrm{cnts}\end{array}$ \\
\hline \multicolumn{9}{|l|}{ E-1 } \\
\hline 182 & 256 & 2065 & $<\mathrm{DL}$ & & $0.041 \pm 0.031$ & 134 & $0.03 \pm 0.01$ & 44 \\
\hline 508 & 263 & 2065 & $0.019 \pm 0.012$ & 38 & $<\mathrm{DL}$ & & $<\mathrm{DL}$ & \\
\hline 1013 & 262 & 2065 & $0.010 \pm 0.009$ & 24 & $<\mathrm{DL}$ & & $0.07 \pm 0.015$ & 78 \\
\hline 1623 & 256 & 2065 & $0.058 \pm 0.013$ & 74 & $<\mathrm{DL}$ & & $0.22 \pm 0.034$ & 134 \\
\hline 2069 & 274 & 2065 & $0.170 \pm 0.024$ & 180 & $0.045 \pm 0.037$ & 344 & $0.23 \pm 0.033$ & 141 \\
\hline \multicolumn{9}{|l|}{ TEW-3 } \\
\hline 101 & 259 & 557 & $<\mathrm{DL}$ & & $<\mathrm{DL}$ & & $0.29 \pm 0.068$ & 125 \\
\hline 303 & 257 & 557 & $0.039 \pm 0.013$ & 37 & $0.065 \pm 0.052$ & 129 & $0.28 \pm 0.037$ & 154 \\
\hline 557 & 252 & 557 & $0.014 \pm 0.015$ & 33 & $0.077 \pm 0.056$ & 213 & $0.37 \pm 0.098$ & 68 \\
\hline \multicolumn{9}{|l|}{ F-L } \\
\hline 101 & 257 & 2670 & $0.008 \pm 0.007$ & 17 & $<\mathrm{DL}$ & & $0.20 \pm 0.130$ & 49 \\
\hline 183 & 260 & 2670 & $<\mathrm{DL}$ & & $0.086 \pm 0.022$ & 246 & $0.19 \pm 0.054$ & 35 \\
\hline 405 & 258 & 2670 & $0.016 \pm 0.009$ & 24 & $<\mathrm{DL}$ & & $0.06 \pm 0.030$ & 22 \\
\hline 907 & 258 & 2670 & $0.039 \pm 0.012$ & 46 & $0.103 \pm 0.060$ & 107 & $0.12 \pm 0.044$ & 28 \\
\hline 1825 & 122 & 2670 & $0.064 \pm 0.017$ & 32 & $0.128 \pm 0.077$ & 239 & $0.54 \pm 0.130$ & 49 \\
\hline 2723 & 124 & 2670 & $0.142 \pm 0.049$ & 123 & $0.265 \pm 0.154$ & 346 & $0.93 \pm 0.097$ & 290 \\
\hline \multicolumn{9}{|l|}{ G-1 } \\
\hline 10 & 269 & 592 & $<\mathrm{DL}$ & & $<\mathrm{DL}$ & & $0.38 \pm 0.042$ & 187 \\
\hline 53 & 251 & 592 & $0.020 \pm 0.009$ & 30 & $0.051 \pm 0.049$ & 107 & $0.31 \pm 0.039$ & 157 \\
\hline 130 & 255 & 592 & $<\mathrm{DL}$ & & $<\mathrm{DL}$ & & $0.26 \pm 0.066$ & 43 \\
\hline 303 & 260 & 592 & $<\mathrm{DL}$ & & $<\mathrm{DL}$ & & $0.38 \pm 0.081$ & 56 \\
\hline 455 & 234 & 592 & $<\mathrm{DL}$ & & $<\mathrm{DL}$ & & $0.29 \pm 0.074$ & 44 \\
\hline 576 & 223 & 592 & $0.088 \pm 0.021$ & 84 & $0.075 \pm 0.058$ & 75 & $0.36 \pm 0.077$ & 48 \\
\hline \multicolumn{9}{|l|}{ E-4W } \\
\hline 94 & 261 & 1385 & $0.020 \pm 0.014$ & 31 & $<\mathrm{DL}$ & & $0.34 \pm 0.079$ & 50 \\
\hline 192 & 260 & 1385 & $<\mathrm{DL}$ & & $<\mathrm{DL}$ & & $0.30 \pm 0.041$ & 135 \\
\hline 608 & 253 & 1385 & $<\mathrm{DL}$ & & $<\mathrm{DL}$ & & $0.29 \pm 0.043$ & 151 \\
\hline 1013 & 123 & 1385 & $<\mathrm{DL}$ & & $0.133 \pm 0.046$ & 93 & $0.43 \pm 0.116$ & 36 \\
\hline 1383 & 123 & 1385 & $0.057 \pm 0.021$ & 25 & $0.071 \pm 0.046$ & 145 & $0.90 \pm 0.164$ & 59 \\
\hline \multicolumn{9}{|l|}{ A3-2 } \\
\hline 101 & 258 & 531 & $<\mathrm{DL}$ & & $<\mathrm{DL}$ & & $0.10 \pm 0.042$ & 25 \\
\hline 152 & 246 & 531 & $<\mathrm{DL}$ & & $<\mathrm{DL}$ & & $0.16 \pm 0.029$ & 115 \\
\hline 233 & 258 & 531 & $<\mathrm{DL}$ & & $<\mathrm{DL}$ & & $0.18 \pm 0.060$ & 33 \\
\hline 303 & 124 & 531 & $<\mathrm{DL}$ & & $<\mathrm{DL}$ & & $0.49 \pm 0.125$ & 39 \\
\hline 404 & 110 & 531 & $<\mathrm{DL}$ & & $<\mathrm{DL}$ & & $0.68 \pm 0.156$ & 44 \\
\hline 518 & 246 & 531 & $0.081 \pm 0.019$ & 58 & $<\mathrm{DL}$ & & $0.70 \pm 0.102$ & 82 \\
\hline \multicolumn{9}{|l|}{ TEW-1 } \\
\hline 82 & 258.5 & 92 & $0.053 \pm 0.015$ & 49 & $0.125 \pm 0.059$ & 334 & $0.88 \pm 0.083$ & 197 \\
\hline \multicolumn{9}{|l|}{ TEW-8 } \\
\hline 20 & 269 & 2800 & $0.011 \pm 0.009$ & 22 & $<\mathrm{DL}$ & 335 & $0.14 \pm 0.032$ & 69 \\
\hline \multicolumn{9}{|l|}{ G-2 } \\
\hline 50 & 229.6 & 67 & $0.094 \pm 0.022$ & 65 & $0.737 \pm 0.097$ & 389 & $0.79 \pm 0.083$ & 167 \\
\hline
\end{tabular}



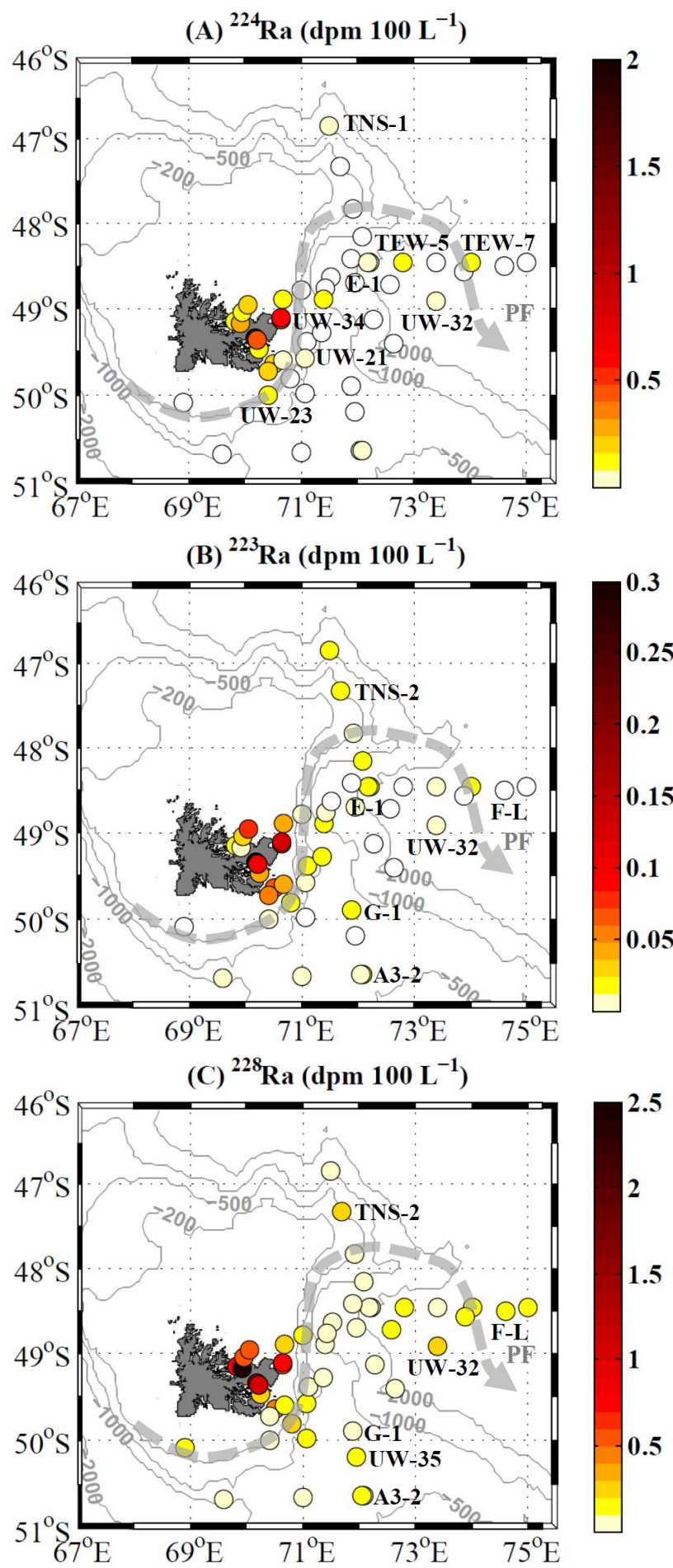

Figure 4. ${ }^{224} \mathrm{Ra}(\mathbf{a}),{ }^{223} \mathrm{Ra}$ (b) and ${ }^{228} \mathrm{Ra}$ (c) distributions in surface waters off the Kerguelen Islands. Radium activities are expressed in $\mathrm{dpm} 100 \mathrm{~L}^{-1}$. White circles indicate samples with Ra activity below the detection limit. A schematic view of the polar front (PF) is shown.
When considering solely the ${ }^{228} \mathrm{Ra}$ vertical profiles which show in most cases an increase in ${ }^{228}$ Ra activities with increasing depth - it cannot be excluded that the vertical mixing contributes to an increase in radium activities in surface waters. However, the ${ }^{224} \mathrm{Ra}$ and ${ }^{223} \mathrm{Ra}$ vertical profiles which show higher Ra activities in the upper and in the deep water column but Ra activities below the detection limit in the mid-water column - clearly indicate that the higher ${ }^{224} \mathrm{Ra}$ and ${ }^{223} \mathrm{Ra}$ activities in surface waters cannot be explained by vertical mixing. The ${ }^{224} \mathrm{Ra}$ and ${ }^{223} \mathrm{Ra}$ enrichments in surface waters are thus more likely explained by the lateral advection of waters that have recently interacted with shallow sediments.

The northward advection of a water mass that has interacted with the shallow sediments deposited on the shelves of Heard Island has been identified as a pathway for the micronutrients that sustain the phytoplankton bloom on the southern Kerguelen Plateau (Chever et al., 2010; van Beek et al., 2008; Zhang et al., 2008). The presence of a chlorophyll plume that expands northward from the southern Kerguelen Plateau may also support the existence of this northward advection (Fig. 2). The observation of significant ${ }^{224} \mathrm{Ra}$ and ${ }^{223} \mathrm{Ra}$ activities in surface waters at station A3-1 confirms this circulation pattern and suggests that the transit time of the waters that interacted with the shelves of Heard Island may be < 1 month between Heard Island and station A-3. This is in agreement with the Ra data obtained in 2005 during the KEOPS- 1 project, where significant ${ }^{224} \mathrm{Ra}$ and ${ }^{223} \mathrm{Ra}$ activities were also found in surface waters at station A3 (van Beek et al., unpublished data). When the waters move further north towards the area investigated in this study, the ${ }^{224} \mathrm{Ra}$ and ${ }^{223} \mathrm{Ra}$ activities will then continue to decay. Two drifters released during the KEOPS- 2 cruise at station A3 allow us to provide constraints on the transit time between the southern Kerguelen Plateau and the studied area (east of Kerguelen at around $49^{\circ} \mathrm{S}$ ). A first drifter recirculated around station A3 nearly 20 days before it moved slowly northward. It took approximately 60-75 days for the drifter to reach the investigated area located to the east of the Kerguelen Islands (Fig. 7). It took approximately 53-65 days for the second drifter to reach the area to the east of Kerguelen. Such transit times agree with the estimate of Park et al. (2008b) during the KEOPS-1 project (i.e., several months between Heard Island and the eastern flank of the Kerguelen Islands). With such a transit time, a water body that interacted with the shelves of Heard Island should not contain any remaining short-lived radium isotopes when reaching the eastern flank of the Kerguelen Islands. As a consequence, the ${ }^{224} \mathrm{Ra}$ and ${ }^{223} \mathrm{Ra}$ activities found in offshore waters east of the Kerguelen Islands, south of the PF, are best explained by diffusion or advection of Ra via waters that recently interacted with the shallow sediments of the northern Kerguelen Plateau. This scenario, however, implies that the $\mathrm{Ra}$ isotopes (and potentially other chemical elements such as iron) were transferred offshore across or via the PF. High dissolved and particulate trace element concen- 
$\bigcirc{ }^{223} \mathrm{Ra}\left(\mathrm{dpm} 100 \mathrm{~L}^{-1}\right) \bigcirc \quad{ }^{224} \mathrm{Ra}\left(\mathrm{dpm} 100 \mathrm{~L}^{-1}\right) \bigcirc \quad{ }^{228} \operatorname{Ra}\left(\mathrm{dpm} 100 \mathrm{~L}^{-1}\right)$
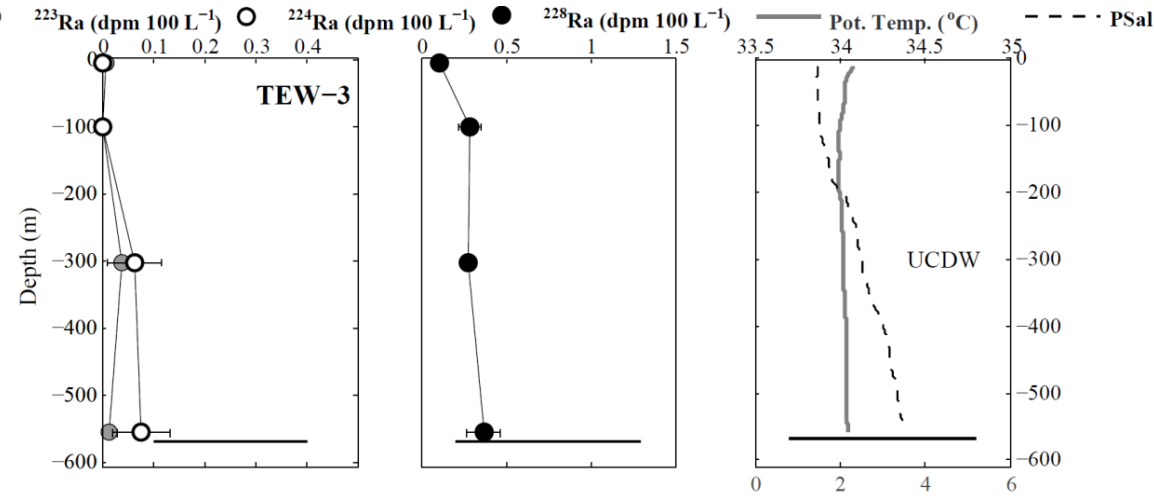

${ }^{223} \mathrm{Ra}\left(\mathrm{dpm} 100 \mathrm{~L}^{-1}\right) \bigcirc \quad{ }^{224} \mathrm{Ra}\left(\mathrm{dpm} 100 \mathrm{~L}^{-1}\right) \bullet{ }^{228} \mathrm{Ra}\left(\mathrm{dpm} 100 \mathrm{~L}^{-1}\right)$
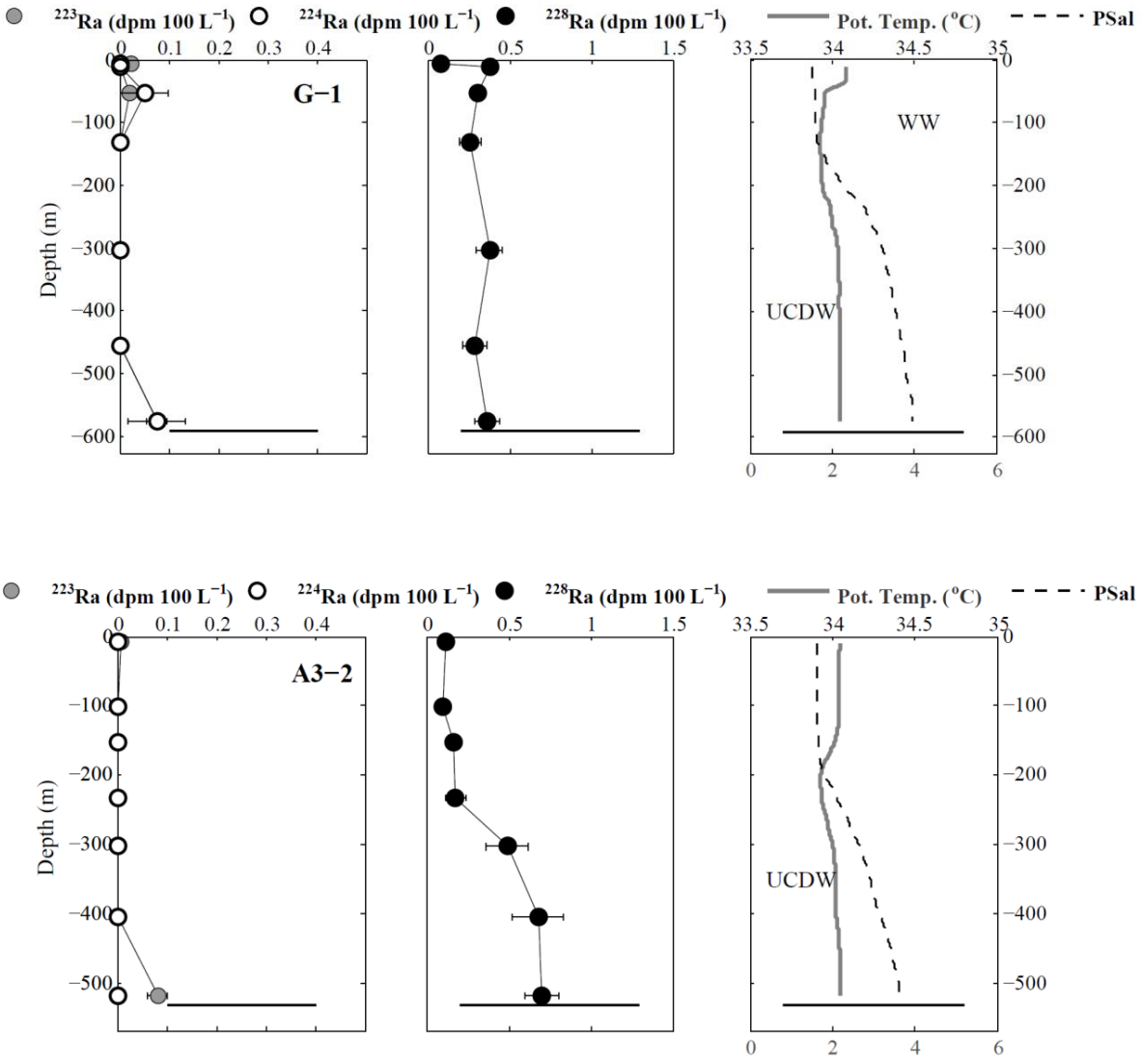

Figure 5. Vertical profiles of ${ }^{223} \mathrm{Ra},{ }^{224} \mathrm{Ra}$ and ${ }^{228} \mathrm{Ra}$ activities $\left(\mathrm{dpm} 100 \mathrm{~L}^{-1}\right)$ at the shallow stations. The main water masses are indicated: Winter Water (WW) and Upper Circumpolar Deep Water (UCDW). The bottom depth is denoted by the horizontal lines.

trations ( $\mathrm{Fe}, \mathrm{Ni}$ and $\mathrm{Co}$ ) were also found east of the PF, confirming that chemical elements may be transported offshore across or via the PF (Quéroué et al. 2014; van der Merwe et al., 2014). Among the potential mechanisms allowing surface waters to be transported eastward across the PF, one can invoke either (i) the wind stress (eastward winds are especially strong in that region) or (ii) eddies that form along the $\mathrm{PF}$ and that could promote the passage of chemical elements across the front.
However, a contribution of surface waters originating from the southern Kerguelen Plateau may not be completely excluded. In contrast to ${ }^{224} \mathrm{Ra}$ and ${ }^{223} \mathrm{Ra}$, which both disappear due to radioactive decay along the northward transport (>2 months), ${ }^{228} \mathrm{Ra}$ with a longer half-life would remain in these waters. The ${ }^{228} \mathrm{Ra}$ activities observed to the east of the Kerguelen Islands may thus be partly explained by an advective transport of waters originating from the south. It cannot be excluded, therefore, that the northward advection origi- 

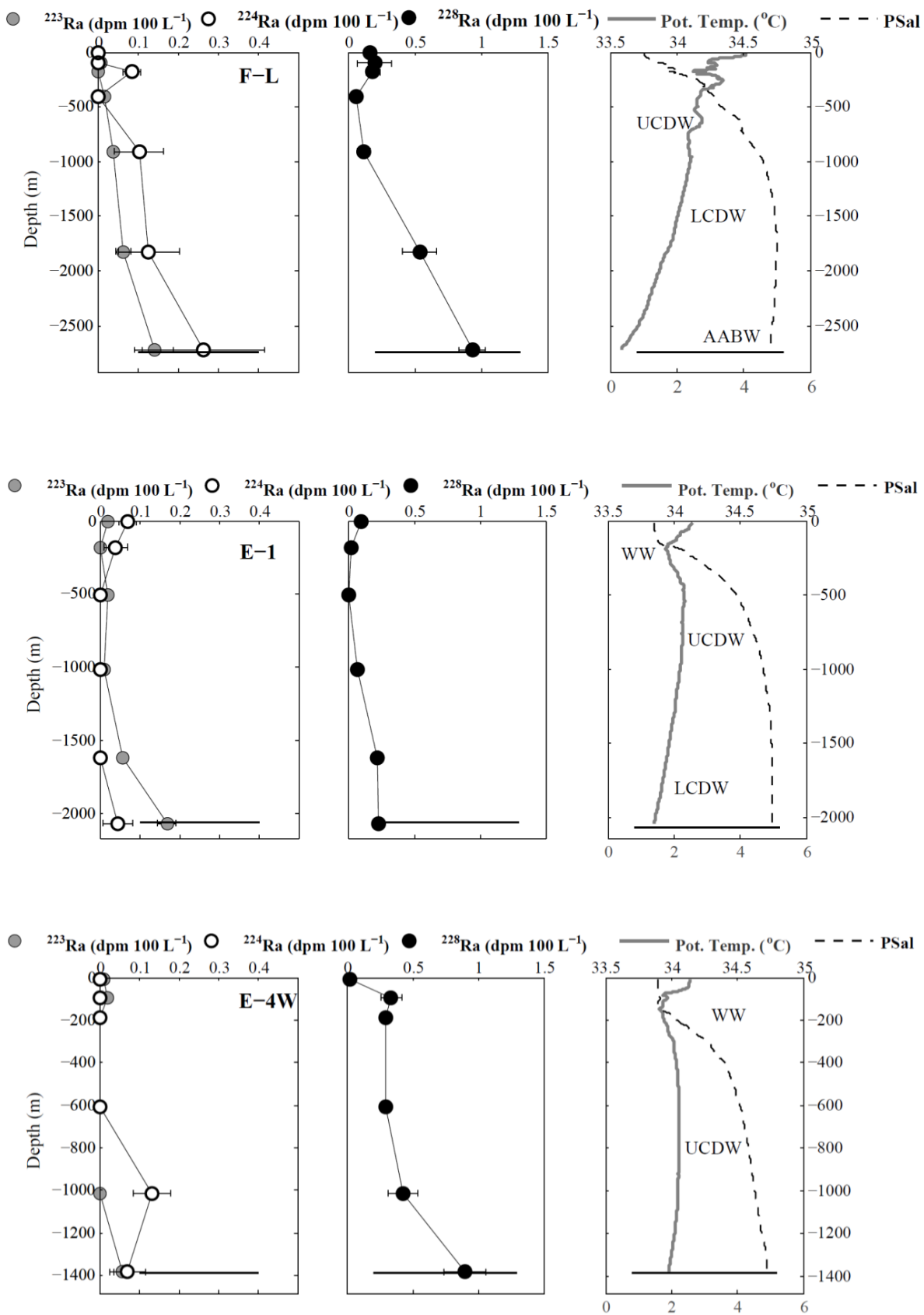

Figure 6. ${ }^{223} \mathrm{Ra},{ }^{224} \mathrm{Ra}$ and ${ }^{228} \mathrm{Ra}$ activities $\left(\mathrm{dpm} 100 \mathrm{~L}^{-1}\right)$ at the deep stations. The major water masses are indicated: Winter Water (WW), Upper Circumpolar Deep Water (UCDW), Lower Circumpolar Deep Water (LCDW) and Antarctic Bottom Water (AABW). The bottom depth is denoted by the horizontal lines.

nating from the southern plateau contributes to the natural fertilization of the investigated area, in addition to the input of chemical elements across the PF that was shown by the short-lived isotopes.

South of the Kerguelen Islands (i.e., along the PF at stations UW-23 and UW-24 or south of the PF, e.g., at stations UW-15, UW-16, R-2; Fig. 4), it cannot be completely excluded that the observed radium enrichments are partly ex- plained by an input of radium associated with the Leclaire Rise, located west of the Kerguelen Islands at ca. $350 \mathrm{~m}$ depth (Weis and Frey, 2002). Station R-2, which is located east of the Leclaire Rise south of the PF, shows significant ${ }^{223} \mathrm{Ra}$ and ${ }^{224} \mathrm{Ra}$ activities in surface waters. Although these activities are relatively low $\left(0.016\right.$ and $0.057 \mathrm{dpm} 100 \mathrm{~L}^{-1}$, respectively), they suggest that the waters downstream of the Leclaire Rise may be impacted by this topographic feature. 


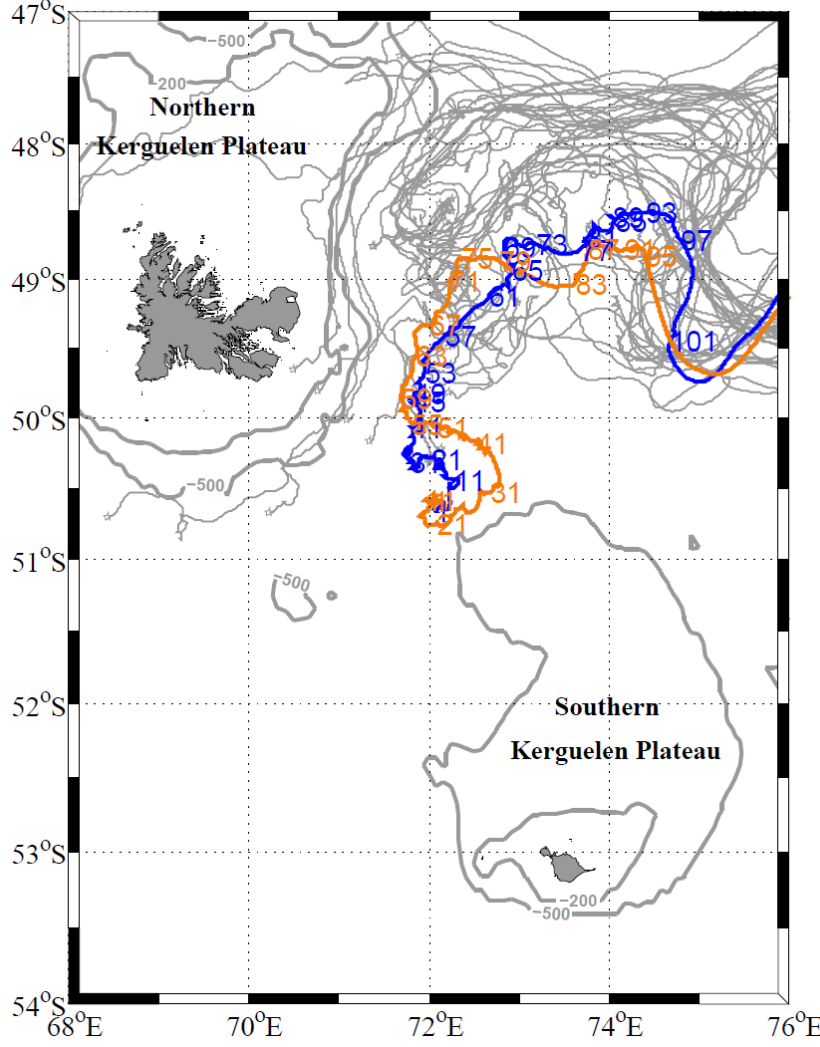

Figure 7. Trajectories of the drifters launched to the east of the Kerguelen Islands during the KEOPS-2 project. The trajectories of the two drifters released at station A3 are reported in color. The equivalent transit time of the two drifters is reported in days along their trajectory. The other drifter trajectories are represented in light gray.

However, sample UW-14 collected in surface waters lying above this rise does not show significant ${ }^{223} \mathrm{Ra}$ and ${ }^{224} \mathrm{Ra}$ activities and only low ${ }^{228} \mathrm{Ra}$ activity, which suggest that vertical mixing may not efficiently transport radium released by the shallow sediments towards surface waters above this topographic feature. Note that the influence of the Leclaire Rise on the chemical element concentrations downstream of the rise is also observed in $\mathrm{Fe}$ and other trace metal (REEs, $\mathrm{Mn}, \mathrm{Al}$ ) concentrations, but only in waters lying in the 200 $500 \mathrm{~m}$ depth interval (Bowie et al., 2014; van der Merwe et al., 2014; Grenier et al., 2015).

\subsection{Timescales of the offshore transport of surface waters}

Once released into the water column, radium isotopes are subject to dilution, mixing and radioactive decay. The decay of short-lived radium isotopes in offshore waters provides information of how quickly chemical elements (including micronutrients) also released by the sediments are diluted and dispersed into the ocean (Moore, 2000). The presence of

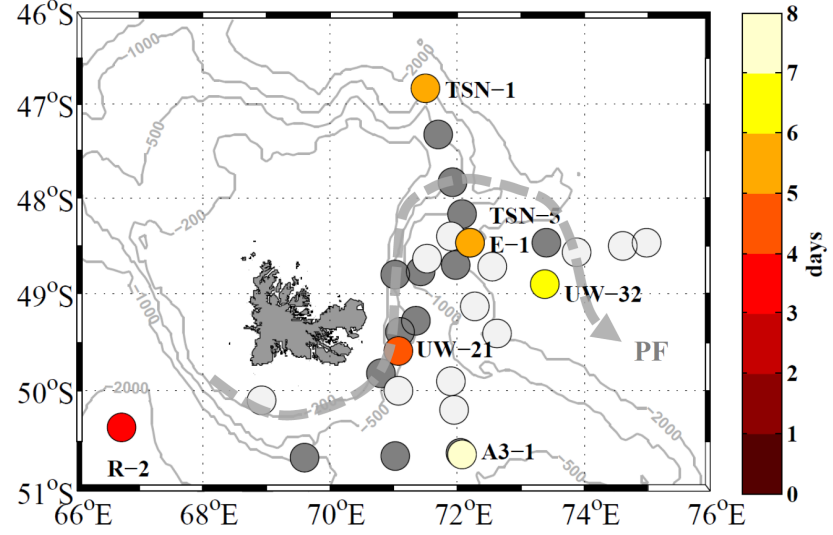

Figure 8. Apparent ages of surface waters determined using the ${ }^{224} \mathrm{Ra} /{ }^{223} \mathrm{Ra}$ ratios. The offshore apparent ages were estimated using an initial ${ }^{224} \mathrm{Ra} /{ }^{223} \mathrm{Ra}$ ratio that was obtained by averaging the ratios found at stations located on the northern Kerguelen Plateau (<200 m water depth). When both ${ }^{224} \mathrm{Ra}$ and ${ }^{223} \mathrm{Ra}$ were significant, apparent ages could be determined (colored symbols). The samples displaying an apparent age between 1 and 2 months are shown in dark gray $\left({ }^{224} \mathrm{Ra}<\mathrm{DL}\right.$ but significant ${ }^{223} \mathrm{Ra}$ activities). Water samples displaying an apparent age $>2$ months are shown in light gray $\left({ }^{224} \mathrm{Ra}<\mathrm{DL}\right.$ and $\left.{ }^{223} \mathrm{Ra}<\mathrm{DL}\right)$. A schematic view of the polar front $(\mathrm{PF})$ is represented.

${ }^{224} \mathrm{Ra}$ and ${ }^{223} \mathrm{Ra}$ in offshore waters thus indicates that the waters have recently been in interaction with the sediments. In contrast, when both ${ }^{224} \mathrm{Ra}$ and ${ }^{223} \mathrm{Ra}$ activities are below the detection limit, this suggests that the water bodies have not been in contact with the sediments over the past 2 months (this is represented in light gray in Fig. 8). The water samples that display significant ${ }^{223} \mathrm{Ra}$ activity but no ${ }^{224} \mathrm{Ra}$ (represented in dark gray in Fig. 8) suggest that the interaction between the water body and the sediment occurred between 1 month $\left({ }^{224} \mathrm{Ra}\right.$ activities <DL) and 2 months ago (significant ${ }^{223} \mathrm{Ra}$ activities). When both the ${ }^{224} \mathrm{Ra}$ and ${ }^{223} \mathrm{Ra}$ activities were significant, apparent ages could be calculated following Moore (2000):

$t=\ln \frac{\left[\frac{224}{223} \mathrm{Ra}\right]_{i}}{\left[\frac{224 \mathrm{Ra}}{223}\right]_{\mathrm{obs}}} * \frac{1}{\lambda_{224}-\lambda_{223}}$,

where $\left({ }^{224} \mathrm{Ra} /{ }^{223} \mathrm{Ra}\right)_{i}$ is the initial ratio in source waters, $\left({ }^{224} \mathrm{Ra} /{ }^{223} \mathrm{Ra}\right)_{\text {obs }}$ is the ratio for a given water sample, and $\lambda_{224}$ and $\lambda_{223}$ are the decay constants of ${ }^{224} \mathrm{Ra}$ and ${ }^{223} \mathrm{Ra}$, respectively. The assumptions inherent to this equation can be found in Moore (2000) and are that (1) the ${ }^{223} \mathrm{Ra}$ and ${ }^{224} \mathrm{Ra}$ activities are constant in the source region (i.e., a constant initial ${ }^{224} \mathrm{Ra} /{ }^{223} \mathrm{Ra}$ ratio is assumed), (2) the ${ }^{224} \mathrm{Ra} /{ }^{223} \mathrm{Ra}$ ratio changes are only due to radioactive decay, and (3) open ocean waters contain no excess ${ }^{223} \mathrm{Ra}$ and ${ }^{224} \mathrm{Ra}$.

In this study, we only reported the apparent ages deduced from the ${ }^{224} \mathrm{Ra} /{ }^{223} \mathrm{Ra}$ ratios because we showed that both 
the ${ }^{224} \mathrm{Ra}$ and ${ }^{223} \mathrm{Ra}$ determined east of Kerguelen originate from the shallow sediments of the Kerguelen Islands (see Sect. 4.1). Apparent ages were thus calculated using an initial ${ }^{224} \mathrm{Ra} /{ }^{223} \mathrm{Ra}$ ratio that was obtained by averaging the ratios found at stations located on the northern Kerguelen Plateau ( $<200 \mathrm{~m}$ water depth). In contrast, we cannot exclude that ${ }^{228} \mathrm{Ra}$ has various origins (Kerguelen Islands and/or Heard Island). The use of the ${ }^{224} \mathrm{Ra} /{ }^{228} \mathrm{Ra}$ or ${ }^{223} \mathrm{Ra} /{ }^{228} \mathrm{Ra}$ ratios to derive apparent ages is thus compromised because it is not possible to determine a single initial ratio in this case.

Several offshore samples display a young apparent age (48 days), suggesting a rapid transport of radium between the shallow waters of the northern Kerguelen Plateau and offshore. Station TNS1, located north of the PF, is reached after 5 days. This observation agrees with the circulation pattern in this area, with waters flowing eastward and that may interact with the shallow northern Kerguelen Plateau (Park et al., 2014). This is also in agreement with the drifters launched during the KEOPS 2 project that also highlighted such advection along the PF (Fig. 7) (Zhou et al., 2014). Station UW-21, located ca. $50 \mathrm{~km}$ offshore, and station E1 and station UW32 , located ca. $200 \mathrm{~km}$ offshore, also show relatively young apparent ages (4, 5 and 6 days, respectively). Because all these stations are located south of the PF, this suggests that the sediment-derived inputs may be rapidly transferred towards offshore waters across the PF. Station R-2, located south of the PF, also displays a young apparent age. At station Kerfix located close to station R-2, Jeandel et al. (1998) reported westward currents associated with a recirculation pattern that may transport chemical elements originating from the Kerguelen Plateau. The Ra signal may then be transported eastward, as suggested by the significant ${ }^{223} \mathrm{Ra}$ activities also observed east of station R-2, south of the PF (Figs. 4 and 8). Alternatively, the Leclaire Rise, located west of R-2, may impact the surface waters, thus leading to a young age for this water sample. Because the ${ }^{224} \mathrm{Ra}$ and ${ }^{223} \mathrm{Ra}$ activities found at station A3-1 were attributed to the northward advection on the southern Plateau, the apparent age at station A3-1 was calculated assuming that the initial ${ }^{224} \mathrm{Ra} /{ }^{223} \mathrm{Ra}$ ratio off Heard Island is similar to that off Kerguelen Islands (Fig. 8). This hypothesis may be correct since the geological contexts of the two islands are similar. The apparent age thus calculated provides an estimate for the transit time of surface waters above the southern Kerguelen Plateau between Heard Island and station A3. However, during the second visit to station A3 (A3-2), the ${ }^{224} \mathrm{Ra}$ and ${ }^{223} \mathrm{Ra}$ activities were below the detection limit. This may highlight the temporal variability in the circulation patterns in this area: the transit time of surface waters between Heard Island and station A3 may thus vary with time, ranging from 1 week to 1-2 months. On such timescales, the ${ }^{228} \mathrm{Ra}$ activities do not significantly decay, which would explain why similar ${ }^{228} \mathrm{Ra}$ activities were found during the two visits to A3. Finally, the spatial variability in the distribution of the apparent ages in offshore waters suggests that the passage of chemical elements across the PF

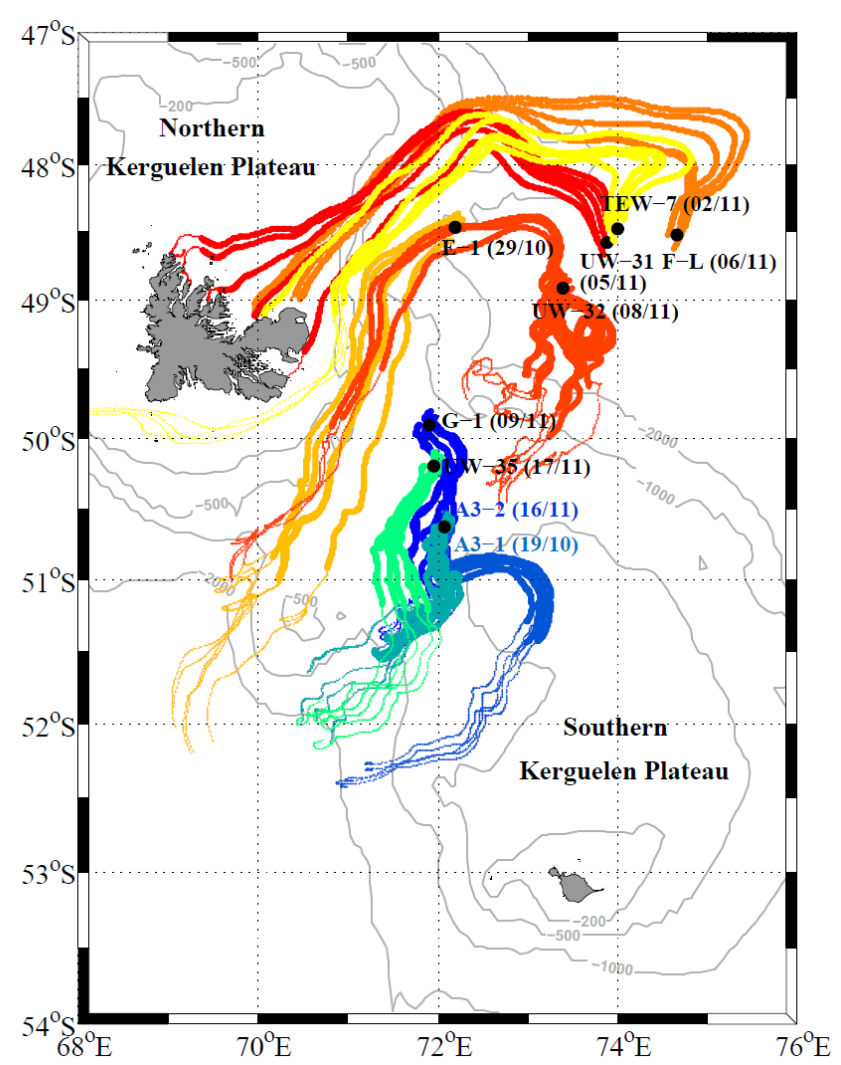

Figure 9. Lagrangian particle analysis derived from total surface currents (considering absolute geostrophic plus Ekman currents). Solid circles represent the location of the stations. The targets for Lagrangian analysis were centered on and around the station locations (to account for spatial variability). The sampling date is indicated in parentheses. Two-month backward trajectories are shown. The first month of the backward trajectory is represented by a thick line. The second month of the backward trajectory is represented by a thinner line.

is a sporadic process, which may contribute to explaining the mosaic structure of the phytoplankton bloom. Future studies in the area could aim to track more precisely the sedimentary sources of radium (and other chemical elements) and to quantify the radium fluxes out of the sediments using, for example, the method developed by Cai et al. (2012).

\subsection{Lagrangian particle analysis}

To provide additional constraints on the origin of the Ra signal in offshore waters, Lagrangian analyses derived from total surface currents were conducted at several stations (Fig. 9). A 2-month backward analysis - to account for the life time of ${ }^{223} \mathrm{Ra}$ - was performed starting from the sampling date for targets that were centered on the station locations. 
The Lagrangian analyses for the southern stations A3-1, A3-2, UW-35 and G-1 are reported in Fig. 9. The backward trajectories provide a similar pattern and indicate a southern origin for the surface waters found at these stations. This pattern agrees with the trajectories of the two drifters released in situ at station A3 (Fig. 7). Waters that have interacted with the shallow shelves of the southern Kerguelen Plateau (Heard Island) may thus reach the investigated area. In particular, this northward advection may explain the significant $\mathrm{Ra}$ activities determined at stations G-1 and A3-1. Note that the distance covered by the backward trajectories over the 2 months is short, thus reflecting the relatively slow currents in this area.

Lagrangian analyses were also performed for several northern stations: E-1, UW-31, UW-32, TEW-7 and F-L. The trajectories are represented in shades of red in Fig. 9. Stations F-L, TEW-7 and UW-31 are located relatively close to each other, east of the Kerguelen Islands in the area of the southern branch of the cyclonic meander formed by the PF. Their backward trajectories display a similar feature and all point to the same origin, which is the northern Kerguelen Plateau. This suggests that chemical elements originating from the Kerguelen Plateau may be transported offshore via the PF. The transit time given by the Lagrangian analysis is approximately 1 month between the coast of the Kerguelen Islands and the investigated stations. With a transit time of approximately 1 month, the ${ }^{224} \mathrm{Ra}$ activities should have disappeared due to radioactive decay - or should be close to the detection limit - while the ${ }^{223} \mathrm{Ra}$ activities should have significantly decayed. As a comparison, the ${ }^{224} \mathrm{Ra}$ and ${ }^{223} \mathrm{Ra}$ activities are below the detection limit at stations F-L and UW-31, whereas significant ${ }^{223} \mathrm{Ra}$ and ${ }^{224} \mathrm{Ra}$ activities were found at station TEW-7. Such a discrepancy between the investigated stations may highlight the spatial variability in the circulation patterns in this area or that the $\mathrm{Ra}$ activities are close to the detection limits. Both ${ }^{224} \mathrm{Ra}$ and ${ }^{223} \mathrm{Ra}$ activities are also significant at station E-1 located in the center of the cyclonic meander formed by the PF. The Lagrangian analysis suggests that the surface waters at station E-1 originate from the southwest. These waters flow northwards before reaching the PF area and then follow the eastern shelf of the northern Kerguelen Plateau. When passing close to the $\mathrm{PF}$, these waters may receive significant Ra inputs (and potentially other sediment-derived inputs) that could be transported via or across the front in this area. This Ra signal may then be transferred to station E-1, as suggested by the backward trajectories. This hypothesis is also supported by the study conducted by Zhou et al. (2014), who identified a northeastward drift of surface waters originating from the Kerguelen Plateau. Finally, the backward trajectories at station UW-32 - which also displayed significant ${ }^{223} \mathrm{Ra}$ and ${ }^{224} \mathrm{Ra}$ - highlight the spatial variability in that area: while several trajectories originate from the south, several other trajectories follow the PF and the shelves of the northern Ker-

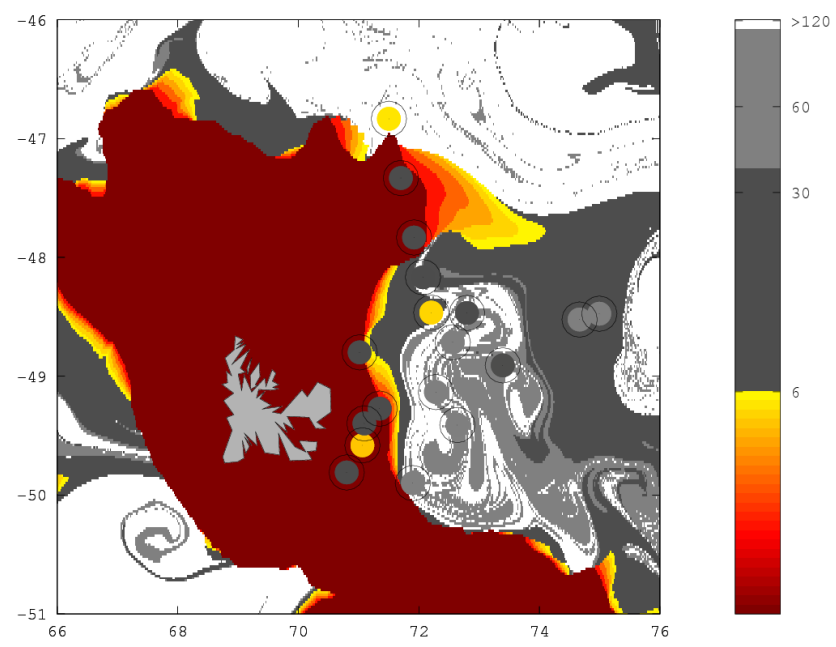

Figure 10. Ages of surface waters (in days) derived from an altimetry Lagrangian-based model. In situ ages derived from radium isotopes are represented by circles. The color bar indicates the time elapsed since the water body left the $2000 \mathrm{~m}$ isobath.

guelen Plateau, where these waters could also potentially receive sediment-derived inputs.

\subsection{Comparison of the apparent radium ages with an altimetry-based Lagrangian model}

The timescale of the offshore transport of surface waters was also investigated using an altimetry-based Lagrangian model (Fig. 10). The color bar indicates the time (number of days) elapsed since the water body left the $2000 \mathrm{~m}$ isobath. A color code similar to that of Fig. 8 was used. A color palette from red to yellow highlights the rapid offshore transport of the surface waters (surface waters $<6$ days). The dark-gray coding illustrates surface waters that left the $2000 \mathrm{~m}$ isobath less than 1 month ago. Finally, surface waters that left the $2000 \mathrm{~m}$ isobath more than 1 month ago are represented in light gray. As a comparison, the radium apparent ages are reported on the map using the same color code.

Young ages can be found close to the $2000 \mathrm{~m}$ isobath, along the PF. Surface waters $<1$ month follow the cyclonic meander formed by the PF, while waters older than 2 months are found in the center of the meander. Note that the altimetry-derived Lagrangian analysis may misplace structures with errors of $\sim 10 \mathrm{~km}$ (e.g., d'Ovidio et al., 2010), which is comparable to the width of the structures visible in the map. It may thus be difficult to compare quantitatively the altimetry-derived ages with the ages determined in situ. Nevertheless, two important considerations can be made: (i) the order of magnitude of the satellite-derived and in situ ages are consistent in the region, and (ii) considering a west-east transect from Kerguelen, both estimations indicate a transition from young to old and then once again young ages, 
which is consistent with the existence of a retentive recirculation region centered at about $73^{\circ} \mathrm{W}, 49^{\circ} \mathrm{S}$.

\section{Conclusions}

The observation of short-lived Ra isotopes $\left({ }^{223} \mathrm{Ra}\right.$ and $\left.{ }^{224} \mathrm{Ra}\right)$ in surface waters east of the Kerguelen Islands, south of the PF, clearly indicates that these waters have recently interacted with shallow sediments. Neither the shelves of Heard Island - located hundreds kilometers south of the study area - nor the vertical mixing of deep waters that interacted with bottom sediments can account for the short-lived radium enrichments found in surface waters. The ${ }^{223} \mathrm{Ra}$ and ${ }^{224} \mathrm{Ra}$ activities south of the PF are thus best explained by waters that interacted with the shelves of the Kerguelen Islands. This finding implies that chemical elements can be transported across or via the PF. Among the potential mechanisms allowing surface waters to be transported eastward across the PF, one can invoke either (i) the wind stress (eastward winds are especially strong in that region) or (ii) eddies that form along the PF and that may promote the transport of surface waters and associated chemical elements. The spatial variability observed in the ${ }^{223} \mathrm{Ra}$ and ${ }^{224} \mathrm{Ra}$ distribution in surface waters south of the PF suggests that the input of waters and associated chemical elements across the $\mathrm{PF}$ - potentially driven by wind stress or eddies - act as sporadic pulses that may highly vary in both space and time. This pathway may thus constitute a mechanism that contributes to fertilizing the phytoplankton bloom with iron and other micronutrients east of the Kerguelen Islands, south of the PF. This finding shows that the PF may not act as a strong barrier for surface waters and associated chemical elements, a finding that may also apply to other frontal systems of the world's ocean.

Author contributions. P. van Beek and B. Lansard performed the sample collection onboard the R/V Marion Dufresne. The sample analysis was done on the ship by P. van Beek and B. Lansard and in the laboratory by V. Sanial and M. Souhaut. F. d'Ovidio developed the model code and E. Kestenare performed the CTD analysis and the simulations for the Lagrangian analysis. M. Zhou provided the drifter data. S. Blain is PI of the KEOPS-2 project and helped interpret the data. V. Sanial interpreted the data and prepared the manuscript.

Acknowledgements. The authors would like to thank Bernard Quéguiner, chief scientist of the KEOPS-2 cruise. We thank the captain and crew members of the R/V Marion Dufresne (IPEVTAAF). We are especially grateful to Pierre Sangiardi (IPEV), who designed the clean pump that allowed us to collect surface water samples in this project. The altimeter current and color/temperature products for the Kerguelen area were produced by Ssalto/Duacs and CLS with support from CNES. This work was funded by the French Research program of INSU-CNRS LEFE-CYBER (Les enveloppes fluides et l'environnement - Cycles biogéochimiques, environnement et ressources), ANR (Agence Nationale de la Recherche, SIMI-6 program, ANR-10-BLAN-0614), CNES (Centre National d'Etudes Spatiales) and IPEV (Institut Polaire Paul-Emile Victor).

Edited by: T. Trull

\section{References}

Annett, A. L., Henley, S. F., Van Beek, P., Souhaut, M., Ganeshram, R., Venables, H. J., Meredith, M. P., and Geibert, W.: Use of radium isotopes to estimate mixing rates and trace sediment inputs to surface waters in northern Marguerite Bay, Antarctic Peninsula, Antarct. Sci., 25, 445-456, doi:10.1017/S0954102012000892, 2013

Arrigo, K. R., van Dijken, G. L., and Bushinsky, S.: Primary production in the Southern Ocean, 1997-2006, J. Geophys. Res.Oceans, 113, C08004, doi:10.1029/2007JC004551, 2008.

Belkin, I. M. and Gordon, A. L.: Southern Ocean fronts from the Greenwich meridian to Tasmania, J. Geophys. Res.-Oceans, 101, 3675-3696, doi:10.1029/95JC02750, 1996.

Blain, S., Tréguer, P., Belviso, S., Bucciarelli, E., Denis, M., Desabre, S., Fiala, M., Martin Jézéquel, V., Le Fèvre, J., Mayzaud, P., Marty, J.-C., and Razouls, S.: A biogeochemical study of the island mass effect in the context of the iron hypothesis: Kerguelen Islands, Southern Ocean, Deep Sea Res. Pt. I, 48, 163-187, doi:10.1016/S0967-0637(00)00047-9, 2001.

Blain, S., Quéguiner, B., Armand, L., Belviso, S., Bombled, B., Bopp, L., Bowie, A., Brunet, C., Brussaard, C., Carlotti, F., Christaki, U., Corbière, A., Durand, I., Ebersbach, F., Fuda, J.L., Garcia, N., Gerringa, L., Griffiths, B., Guigue, C., Guillerm, C., Jacquet, S., Jeandel, C., Laan, P., Lefèvre, D., Lo Monaco, C., Malits, A., Mosseri, J., Obernosterer, I., Park, Y.-H., Picheral, M., Pondaven, P., Remenyi, T., Sandroni, V., Sarthou, G., Savoye, N., Scouarnec, L., Souhaut, M., Thuiller, D., Timmermans, K., Trull, T., Uitz, J., Van Beek, P., Veldhuis, M., Vincent, D., Viollier, E., Vong, L., and Wagener, T.: Effect of natural iron fertilization on carbon sequestration in the Southern Ocean, Nature, 446, 10701074, doi:10.1038/nature05700, 2007.

Bowie, A. R., van der Merwe, P., Quéroué, F., Trull, T., Fourquez, M., Planchon, F., Sarthou, G., Chever, F., Townsend, A. T., Obernosterer, I., Sallée, J.-B., and Blain, S.: Iron budgets for three distinct biogeochemical sites around the Kerguelen archipelago (Southern Ocean) during the natural fertilisation experiment KEOPS-2, Biogeosciences Discuss., 11, 17861-17923, doi:10.5194/bgd-11-17861-2014, 2014.

Cai, P., Shi, X., Moore, W. S., and Dai, M.: Measurement of ${ }^{224} \mathrm{Ra}: 228 \mathrm{Th}$ disequilibrium in coastal sediments using a delayed coincidence counter, Mar. Chem., 138-139, 1-6, doi:10.1016/j.marchem.2012.05.004, 2012.

Charette, M. A., Buesseler, K. O., and Andrews, J. E.: Utility of radium isotopes for evaluating the input and transport of groundwater-drived nitrogen to a Cape Cod estuary, Limnol. Oceanogr., 46, 465-470, 2001.

Charette, M. A., Gonneea, M. E., Morris, P. J., Statham, P., Fones, G., Planquette, H., Salter, I., and Garabato, A. N.: Radium isotopes as tracers of iron sources fueling a Southern Ocean 
phytoplankton bloom, Deep Sea Res. Pt. II, 54, 1989-1998, doi:10.1016/j.dsr2.2007.06.003, 2007.

Charrassin, J.-B., Park, Y.-H., Le Maho, Y., and Bost, C.-A.: Fine resolution 3D temperature fields off Kerguelen from instrumented penguins, Deep Sea Res. Pt. I, 51, 2091-2103, doi:10.1016/j.dsr.2004.07.019, 2004.

Chever, F., Sarthou, G., Bucciarelli, E., Blain, S., and Bowie, A. R.: An iron budget during the natural iron fertilisation experiment KEOPS (Kerguelen Islands, Southern Ocean), Biogeosciences, 7, 455-468, doi:10.5194/bg-7-455-2010, 2010.

De Baar, H. J. W., De Jong, J. T. M., Bakker, D. C. E., Löscher, B. M., Veth, C., Bathmann, U., and Smetacek, V.: Importance of iron for plankton blooms and carbon dioxide drawdown in the Southern Ocean, Nature, 373, 412-415, doi:10.1038/373412a0, 1995.

Dibarboure, G., Pujol, M.-I., Briol, F., Traon, P. Y. L., Larnicol, G., Picot, N., Mertz, F., and Ablain, M.: Jason-2 in DUACS: Updated System Description, First Tandem Results and Impact on Processing and Products, Mar. Geod., 34, 214-241, doi:10.1080/01490419.2011.584826, 2011.

d'Ovidio, F., De Monte, S., Alvain, S., Dandonneau, Y., and Levy, M.: Fluid dynamical niches of phytoplankton types, P. Natl. Acad. Sci. USA, 107, 18366-18370, doi:10.1073/pnas.1004620107, 2010.

Dulaiova, H., Ardelan, M. V., Henderson, P. B., and Charette, M. A.: Shelf-derived iron inputs drive biological productivity in the southern Drake Passage, Glob. Biogeochem. Cy., 23, GB4014, doi:10.1029/2008GB003406, 2009.

Emery, W. J. and Meincke, J.: Global water masses: summary and review, Oceanol. Acta, 9, 383-391, 1986.

Garcia-Solsona, E., Garcia-Orellana, J., Masqué, P., and Dulaiova, H.: Uncertainties associated with ${ }^{223} \mathrm{Ra}$ and ${ }^{224} \mathrm{Ra}$ measurements in water via a Delayed Coincidence Counter (RaDeCC), Mar. Chem., 109, 198-219, doi:10.1016/j.marchem.2007.11.006, 2008.

Grenier, M., Garcia-Solsona, E., Lemaitre, N., Bouvier, V., Nonnotte, P., and Jeandel, C.: Differentiating lithogenic supplies, water mass transport and biological processes on and off the Kerguelen Plateau using the rare earth data, in preparation, 2015.

Hanfland, C.: Radium-226 and Radium-228 in the Atlantic Sector of the Southern Ocean, thesis, Alfred Wegener Institute, Bremerhaven, Allemagne, 2002.

Jeandel, C., Ruiz-Pino, D., Gjata, E., Poisson, A., Brunet, C., Charriaud, E., dehairs, F., delille, D., Fiala, M., Fravalo, C., Carlos Miquel, J., Park, Y., Pondaven, P., Queguiner, B., Razouls, S., Shauer, B., and Treguer, P.: KERFIX, a time-series station in the Southern Ocean: a presentation, J. Mar. Syst., 17, 555-569, doi:10.1016/S0924-7963(98)00064-5, 1998.

Kaufman, A., Trier, R. M., Broecker, W. S., and Feely, H. W.: Distribution of ${ }^{228} \mathrm{Ra}$ in the world ocean, J. Geophys. Res., 78, 88278848, doi:10.1029/JC078i036p08827, 1973.

Korb, R. E., Whitehouse, M. J., and Ward, P.: SeaWiFS in the southern ocean: spatial and temporal variability in phytoplankton biomass around South Georgia, Deep Sea Res. Pt. II, 51, 99-116, doi:10.1016/j.dsr2.2003.04.002, 2004.

Martin, J. H., Fitzwater, S. E., and Gordon, R. M.: Iron deficiency limits phytoplankton growth in Antarctic waters, Global Biogeochem. Cy., 4, 5-12, doi:10.1029/GB004i001p00005, 1990.
Mongin, M. M., Abraham, E. R., and Trull, T. W.: Winter advection of iron can explain the summer phytoplankton bloom that extends $1000 \mathrm{~km}$ downstream of the Kerguelen Plateau in the Southern Ocean, J. Mar. Res., 67, 225-237, doi:10.1357/002224009789051218, 2009.

Moore, J. K. and Abbott, M. R.: Surface chlorophyll concentrations in relation to the Antarctic Polar Front: seasonal and spatial patterns from satellite observations, J. Mar. Syst., 37, 69-86, doi:10.1016/S0924-7963(02)00196-3, 2002.

Moore, W. S.: Ages of continental shelf waters determined from ${ }^{223} \mathrm{Ra}$ and ${ }^{224} \mathrm{Ra}$, J. Geophys. Res., 105, 22117, doi:10.1029/1999JC000289, 2000.

Moore, W. S.: Fifteen years experience in measuring ${ }^{224} \mathrm{Ra}$ and ${ }^{223} \mathrm{Ra}$ by delayed-coincidence counting, Mar. Chem., 109, 188197, doi:10.1016/j.marchem.2007.06.015, 2008.

Moore, W. S. and Arnold, R.: Measurement of ${ }^{223} \mathrm{Ra}$ and ${ }^{224} \mathrm{Ra}$ in coastal waters using a delayed coincidence counter, J. Geophys. Res.-Oceans, 101, 1321-1329, doi:10.1029/95JC03139, 1996.

Orsi, A. H., Whitworth III, T., and Nowlin Jr., W. D.: On the meridional extent and fronts of the Antarctic Circumpolar Current, Deep Sea Res. Pt. I, 42, 641-673, doi:10.1016/09670637(95)00021-W, 1995.

Park, Y.-H. and Gambéroni, L.: Large-scale circulation and its variability in the south Indian Ocean from TOPEX/POSEIDON altimetry, J. Geophys. Res.-Oceans, 100, 24911-24929, doi:10.1029/95JC01962, 1995.

Park, Y.-H. and Gamberoni, L.: Cross-frontal exchange of Antarctic Intermediate Water and Antarctic Bottom Water in the Crozet Basin, Deep Sea Res. Pt. II, 44, 963-986, doi:10.1016/S09670645(97)00004-0, 1997.

Park, Y.-H., Gamberoni, L., and Charriaud, E.: Frontal structure, water masses, and circulation in the Crozet Basin, J. Geophys. Res., 98, 12361, doi:10.1029/93JC00938, 1993.

Park, Charriaud E., and Fieux, M.: Thermohaline structure of the Antarctic Surface Water/Winter Water in the Indian sector of the Southern Ocean, J. Mar. Syst., 17, 5-23, doi:10.1016/S09247963(98)00026-8, 1998.

Park, Y.-H., Charriaud, E., Pino, D. R., and Jeandel, C.: Seasonal and interannual variability of the mixed layer properties and steric height at station KERFIX, southwest of Kerguelen, J. Mar. Syst., 17, 571-586, doi:10.1016/S0924-7963(98)00065-7, 1998b.

Park, Y.-H., Fuda, J.-L., Durand, I., and Naveira Garabato, A. C.: Internal tides and vertical mixing over the Kerguelen Plateau, Deep Sea Res. Pt. II, 55, 582-593, doi:10.1016/j.dsr2.2007.12.027, 2008a.

Park, Y.-H., Roquet, F., Durand, I., and Fuda, J.-L.: Large-scale circulation over and around the Northern Kerguelen Plateau, Deep Sea Res. Pt. II, 55, 566-581, doi:10.1016/j.dsr2.2007.12.030, $2008 b$.

Park, Y.-H., Vivier, F., Roquet, F., and Kestenare, E.: Direct observations of the ACC transport across the Kerguelen Plateau, Geophys. Res. Lett., 36, L18603, doi:10.1029/2009GL039617, 2009

Park, Y.-H., Durand, I., Kestenare, E., Rougier, G., Zhou, M., d'Ovidio, F., Cotté, C., and Lee, J.-H.: Polar Front around the Kerguelen Islands: An up-to-date determination and associated circulation of surface/subsurface waters, J. Geophys. Res.Oceans, 119, 6575-6592, doi:10.1002/2014JC010061, 2014. 
Pollard, R., Lucas, M., and Read, J.: Physical controls on biogeochemical zonation in the Southern Ocean, Deep Sea Res. Pt. II, 49, 3289-3305, doi:10.1016/S0967-0645(02)00084-X, 2002.

Pollard, R., Sanders, R., Lucas, M., and Statham, P.: The Crozet Natural Iron Bloom and Export Experiment (CROZEX), Deep Sea Res. Pt. II, 54, 1905-1914, doi:10.1016/j.dsr2.2007.07.023, 2007.

Quéroué, F., Sarthou, G., Planquette, H. F., Bucciarelli, E., Chever, F., van der Merwe, P., Lannuzel, D., Townsend, A. T., Cheize, M., Blain, S., d'Ovidio, F., and Bowie, A. R.: High variability of dissolved iron concentrations in the vicinity of Kerguelen Island (Southern Ocean), Biogeosciences Discuss., 12, 231-270, doi:10.5194/bgd-12-231-2015, 2015.

Sanial, V., Van Beek, P., Lansard, B., D’ Ovidio, F., Kestenare, E., Souhaut, M., Zhou, M., and Blain, S.: Study of the phytoplankton plume dynamics off the Crozet Islands (Southern Ocean): A geochemical-physical coupled approach, J. Geophys. Res. Oceans, 119, 2227-2237, doi:10.1002/2013JC009305, 2014.

Tagliabue, A., Sallée, J.-B., Bowie, A. R., Lévy, M., Swart, S., and Boyd, P. W.: Surface-water iron supplies in the Southern Ocean sustained by deep winter mixing, Nat. Geosci., 7, 314320, doi:10.1038/ngeo2101, 2014.

van Beek, P., Bourquin, M., Reyss, J.-L., Souhaut, M., Charette, M. A., and Jeandel, C.: Radium isotopes to investigate the water mass pathways on the Kerguelen Plateau (Southern Ocean), Deep Sea Res. Pt. II, 55, 622-637, doi:10.1016/j.dsr2.2007.12.025, 2008.

van Beek, P., Souhaut, M., Lansard, B., Bourquin, M., Reyss, J.L., Von Ballmoos, P., and Jean, P.: LAFARA: a new underground laboratory in the French Pyrenees for ultra low-level gamma-ray spectrometry, J. Environ. Radioactiv., 116, 152-158, doi:10.1016/j.jenvrad.2012.10.002, 2013. van Beek, P., Souhaut, M., and Reyss, J.-L.: Measuring the radium quartet $\left({ }^{228} \mathrm{Ra},{ }^{226} \mathrm{Ra},{ }^{224} \mathrm{Ra},{ }^{223} \mathrm{Ra}\right)$ in seawater samples using gamma spectrometry, J. Environ. Radioactiv., 101, 521-529, doi:10.1016/j.jenvrad.2009.12.002, 2010.

van der Merwe, P., Bowie, A. R., Quéroué, F., Armand, L., Blain, S., Chever, F., Davies, D., Dehairs, F., Planchon, F., Sarthou, G., Townsend, A. T., and Trull, T.: Sourcing the iron in the naturally-fertilised bloom around the Kerguelen Plateau: particulate trace metal dynamics, Biogeosciences Discuss., 11, 1338913432, doi:10.5194/bgd-11-13389-2014, 2014.

Weis, D. and Frey, F. A.: Submarine Basalts of the Northern Kerguelen Plateau: Interaction Between the Kerguelen Plume and the Southeast Indian Ridge Revealed at ODP Site 1140, J. Petrol., 43, 1287-1309, doi:10.1093/petrology/43.7.1287, 2002.

Zhang, Y., Lacan, F., and Jeandel, C.: Dissolved rare earth elements tracing lithogenic inputs over the Kerguelen Plateau (Southern Ocean), Deep Sea Res. Pt. II, 55, 638-652, doi:10.1016/j.dsr2.2007.12.029, 2008.

Zhou, M., Zhu, Y., d'Ovidio, F., Park, Y.-H., Durand, I., Kestenare, E., Sanial, V., Van-Beek, P., Queguiner, B., Carlotti, F., and Blain, S.: Surface currents and upwelling in Kerguelen Plateau regions, Biogeosciences Discuss., 11, 6845-6876, doi:10.5194/bgd-116845-2014, 2014. 